\title{
Mirror symmetry and elliptic Calabi-Yau manifolds
}

\section{Yu-Chien Huang and Washington Taylor}

Center for Theoretical Physics, Department of Physics, Massachusetts Institute of Technology, 77 Massachusetts Avenue, Cambridge, MA 02139, U.S.A.

E-mail: yc huang@mit.edu, wati@mit.edu

ABSTRACT: We find that for many Calabi-Yau threefolds with elliptic or genus one fibrations mirror symmetry factorizes between the fiber and the base of the fibration. In the simplest examples, the generic CY elliptic fibration over any toric base surface $B$ that supports an elliptic Calabi-Yau threefold has a mirror that is an elliptic fibration over a dual toric base surface $\tilde{B}$ that is related through toric geometry to the line bundle $-6 K_{B}$. The Kreuzer-Skarke database includes all these examples and gives a wide range of other more complicated constructions where mirror symmetry also factorizes. Since recent evidence suggests that most Calabi-Yau threefolds are elliptic or genus one fibered, this points to a new way of understanding mirror symmetry that may apply to a large fraction of smooth Calabi-Yau threefolds. The factorization structure identified here can also apply for CalabiYau manifolds of higher dimension.

KEYwords: Differential and Algebraic Geometry, F-Theory, String Duality, Superstring Vacua

ArXiv EPrint: 1811.04947 


\section{Contents}

1 Introduction 1

2 Toric hypersurface Calabi-Yau manifolds, fibrations and mirror symmetry 2

2.1 Toric hypersurfaces and fibrations 3

2.2 Factorization of mirror symmetry 5

3 Generic Calabi-Yau elliptic fibrations over toric base surfaces $\quad 7$

$\begin{array}{lll}3.1 & \text { General case } & 7\end{array}$

3.2 Example: generic elliptic fibration over $\mathbb{P}^{2}$ (Hodge numbers $(2,272)$ ) 9

3.3 Example: self-mirror Calabi-Yau threefold with Hodge numbers $(251,251) \quad 10$

3.4 Example: generic elliptic fibration over $\mathbb{F}_{n}$ (Hodge numbers $(3,243), \ldots$, $(11,491) \quad 11$

4 Some further examples $\quad \mathbf{1 6}$

4.1 Tunings of generic fibrations (example: tuning an $\mathrm{SU}(2)$ on $\mathbb{P}^{2}$ ) 17

$\begin{array}{lll}4.2 & \text { Tunings of generic fibrations over base } B \text { and mirror base } \tilde{B} & 18\end{array}$

$\begin{array}{lll}4.3 & \text { Standard stacking } F_{10} \text {-fibered } \nabla \text { vs. non-standard } F_{10} \text {-fibered } \Delta & 19\end{array}$

4.4 Other toric fibers (example: vertex stacking on fiber $F_{2}=\mathbb{P}^{1} \times \mathbb{P}^{1}$ ) 20

4.5 Elliptic fibration with a non-fibered mirror 22

4.6 Elliptic Calabi-Yau fourfolds (example: generic elliptic fibration over $\mathbb{P}^{3}$ ) 22

5 Conclusions and further questions $\quad 23$

$\begin{array}{lll}5.1 & \text { Summary of results } & 23\end{array}$

5.2 Further questions and directions 24

A The 16 reflexive $2 \mathrm{D}$ fiber polytopes $\nabla_{2} \quad 26$

B Faces of the base polytope and chains of non-Higgsable clusters $\quad 27$

\section{Introduction}

Calabi-Yau manifolds have been a major subject of study in mathematics and physics over the last three decades, following the realization that these geometries can be used to compactify string theory in a way that preserves supersymmetry [1]. One of the most intriguing aspects of Calabi-Yau threefolds is the existence of an equivalence known as mirror symmetry (see e.g. [2]) that relates the physics of a type IIA string compactification on a CalabiYau threefold $X$ to that arising from a type IIB string compactification on a mirror threefold $\tilde{X}$. One of the first important clues to mirror symmetry was the observation $[3,4]$ that the 
Calabi-Yau threefolds generated by hypersurfaces in toric varieties have the property that for every Calabi-Yau threefold $X$ with Hodge numbers $h^{1,1}(X), h^{2,1}(X)$ there is a corresponding mirror threefold $\tilde{X}$ with Hodge numbers $h^{1,1}(\tilde{X})=h^{2,1}(X), h^{2,1}(\tilde{X})=h^{1,1}(X)$. The largest known set of Calabi-Yau threefolds are constructed from the class of over 400 million reflexive $4 \mathrm{D}$ polytopes found by Kreuzer and Skarke [5, 6], and exhibit this mirror symmetry structure.

More recently, an increasing body of evidence [7-16] suggests that a large fraction of known Calabi-Yau threefolds have the property that they can be described as genus one or elliptic fibrations over a complex two-dimensional base surface. We recently showed that this is true of all but at most 4 Calabi-Yau threefolds in the Kreuzer-Skarke database having one or the other Hodge number $h^{2,1}, h^{1,1}$ at least 140 , and that at small $h^{1,1}$ the fraction of polytopes in the Kreuzer-Skarke database that lack an obvious elliptic or genus one fibration decreases roughly as $0.1 \times 2^{5-h^{1,1}}$. In this paper we show that the structure of these fibrations gives a natural way of "factorizing" mirror symmetry for many elliptic and genus one fibered toric hypersurface Calabi-Yau threefolds, so that the fiber of $X$ determines the fiber of the mirror threefold $\tilde{X}$, and the base and fibration structure of $X$ determine the base of $\tilde{X}$. A key aspect of this factorization involves the observation that a simple additional condition on the fibration structure of an elliptic toric hypersurface Calabi-Yau threefold $X$ implies that the mirror $\tilde{X}$ is also elliptic and has a mirror elliptic fiber characterized by a $2 \mathrm{D}$ polytope dual to the one that contains the genus one or elliptic fiber of $X$. Such mirror fibers were also studied in the related context of K3 surfaces and heterotic/F-theory duality in $[8,17-20]$, and in the context of elliptic fibers for Ftheory in [21].

The outline of this paper is as follows: in section 2, we review some basic aspects of toric hypersurface Calabi-Yau manifolds and elliptic and genus one fibrations; we then describe in general the way in which mirror symmetry can factorize for toric hypersurface CalabiYau manifolds. In section 3, we consider the simplest examples of this factorization: when $X$ is the generic CY elliptic fibration over any toric base surface $B$ that supports an elliptic Calabi-Yau threefold, the mirror $\tilde{X}$ has a simple description as an elliptic fibration over a dual base $\tilde{B}$ that has a simple description in toric geometry in terms of $B$. In section 4 , we consider some further examples, including Weierstrass/Tate tunings of generic models over a toric base, "stacked" fibrations with non-generic fiber types, and analogous factorization for elliptic Calabi-Yau fourfolds. We conclude in section 5 with a summary and some open questions.

\section{Toric hypersurface Calabi-Yau manifolds, fibrations and mirror sym- metry}

In this section we review some basic aspects of toric hypersurface Calabi-Yau threefolds and fibrations, and we describe the basic framework of mirror symmetry factorization that applies for many elliptic and genus one fibered toric hypersurface Calabi-Yau manifolds. Much of the material reviewed in the first part of this section is covered in more detail in the papers $[15,16]$. 


\subsection{Toric hypersurfaces and fibrations}

A broad class of Calabi-Yau manifolds can be described as hypersurfaces in toric varieties following the approach of Batyrev [22]. A lattice polytope $\nabla$ is defined to be the set of lattice points in $N=\mathbb{Z}^{n}$ that are contained within the convex hull of a finite set of vertices $v_{i} \in N$. The dual of a polytope $\nabla$ is defined to be

$$
\nabla^{*}=\left\{u \in M_{\mathbb{R}}=M \otimes \mathbb{R}:\langle u, v\rangle \geq-1, \forall v \in \nabla\right\},
$$

where $M=N^{*}=\operatorname{Hom}(N, \mathbb{Z})$ is the dual lattice. A lattice polytope $\nabla \subset N$ containing the origin is reflexive if its dual polytope is also a lattice polytope. For any reflexive polytope, the origin is the unique interior point.

When $\nabla$ is reflexive, we denote the dual polytope by $\Delta=\nabla^{*}$. The elements of the dual polytope $\Delta$ can be associated with monomials in a section of the anti-canonical bundle of a toric variety associated to $\nabla$. A section of this bundle defines a hypersurface in the toric variety associated to $\nabla$; this hypersurface is a Calabi-Yau manifold of dimension $n-1$. The polytopes $\nabla$ and $\Delta$ describe toric hypersurface Calabi-Yau manifolds that are related by mirror symmetry [4]. As $\nabla$ and $\Delta$ are a pair of $4 \mathrm{D}$ reflexive polytopes, there is a one-to-one correspondence between $l$-dimensional faces $\theta$ of $\Delta$ and $(4-l)$-dimensional faces $\tilde{\theta}$ of $\nabla$ related by the dual operation

$$
\theta^{*}=\{y \in \nabla,\langle y, p t\rangle=-1 \mid \text { for all } p t \text { that are vertices of } \theta\} .
$$

For the CY associated with $\nabla$, the Hodge numbers are given by

$$
\begin{aligned}
& h^{2,1}=\operatorname{pts}(\Delta)-\sum_{\theta \in F_{3}^{\Delta}} \operatorname{int}(\theta)+\sum_{\theta \in F_{2}^{\Delta}} \operatorname{int}(\theta) \operatorname{int}\left(\theta^{*}\right)-5, \\
& h^{1,1}=\operatorname{pts}(\nabla)-\sum_{\tilde{\theta} \in F_{3}^{\nabla}} \operatorname{int}(\tilde{\theta})+\sum_{\tilde{\theta} \in F_{2}^{\nabla}} \operatorname{int}(\tilde{\theta}) \operatorname{int}\left(\tilde{\theta}^{*}\right)-5,
\end{aligned}
$$

where $\theta$ are faces of $\Delta, \tilde{\theta}$ are faces of $\nabla, F_{l}^{\nabla / \Delta}$ denotes the set of $l$-dimensional faces of $\nabla$ or $\Delta(l<n)$, and $\operatorname{pts}(\nabla / \Delta):=$ number of lattice points of $\nabla$ or $\Delta, \operatorname{int}(\theta / \tilde{\theta}):=$ number of lattice points interior to $\theta$ or $\tilde{\theta}$. The correspondence (2.2) makes the duality between the Hodge number formulae manifest.

When the polytope $\nabla$ has a $2 \mathrm{D}$ subpolytope $\nabla_{2}$ that is also reflexive, the associated Calabi-Yau manifold has a genus one fibration [23]. ${ }^{1}$ This fibration is characterized by a projection $\pi$ on $N=\mathbb{Z}^{n}$ that maps the fiber $\nabla_{2}$ to 0 . For a 4 D polytope $\nabla$, the base $B$ of the fibration is described by the $2 \mathrm{D}$ toric variety associated with the set of primitive rays in the image of $\nabla$ under the projection $\pi: \mathbb{Z}^{4} \rightarrow \mathbb{Z}^{2}$, where a primitive ray is one that

\footnotetext{
${ }^{1}$ In [24] it is argued that in some cases the condition for a fibration is more subtle; in particular, the clearest way of constructing a fibration uses a toric morphism that must be compatible with a triangulation of $\nabla$, which may not be possible in some cases, particularly in higher dimensions. We do not analyze the detailed structure of triangulations in this paper; for the simple cases considered here there does not seem to be any obstruction to the existence of a triangulation giving a toric morphism compatible with the fibration, but this in principle should be checked in detail, particularly for higher-dimensional varieties where the triangulation of the base is not unique.
} 
is not an integer multiple of another element of the lattice. Since the base in this case is two-dimensional, the triangulation in the resulting toric variety is uniquely determined by the ordering of the rays; in higher dimensions, there may be many distinct triangulations possible.

There are 16 distinct reflexive 2D polytopes, listed in appendix A. The structure of the genus one and elliptic fibrations associated with each of these 16 fibers is studied in some detail in [25] and in the F-theory context in [16, 21, 26, 27]. As discussed in these papers, all fibers other than $F_{1}, F_{2}, F_{4}$ contain at least one curve of self-intersection -1 ; such a curve gives rise to a global section so that the fibration is elliptic and not just genus one. Associated with each of the 16 reflexive 2D polytopes $F=\nabla_{2}=F_{i}$ is a dual fiber $\tilde{F}$, given by $\tilde{F}_{i}=\Delta_{2}=F_{17-i}$ for all $i$ except $i=7,8,9,10$ in which cases $\tilde{F}_{i} \cong F_{i}$ under a linear change of coordinates. We will refer to $\tilde{F}$ as the mirror fiber of $F$. The anticanonical hypersurfaces in $F, \tilde{F}$ represent a mirror pair of 1D Calabi-Yau varieties (genus one curves). Aspects of these dual fibers and associated mirror curves have previously been encountered and studied in the contexts of K3 fibrations, heterotic/F-theory duality and F-theory fibers in $[8,17-21]$.

When a polytope $\nabla$ admits a toric fibration of this kind, the lattice points in the dual polytope $\Delta$ can be associated with monomials in various line bundles over the base $B$. We can choose a coordinate system on $N$ so that the vertices of the fiber $F=\nabla_{2}$ lie in the plane $(0,0 ; \cdot, \cdot)$. Each lattice point $v \in \nabla$ can then be represented in the form

$$
v=\left(v_{1}, v_{2} ; v_{3}, v_{4}\right)=\left(v^{(I)} ; v^{(I I)}\right),
$$

where the first two coordinates $v^{(I)}=\left(v_{1}, v_{2}\right)$ correspond to the base direction, and the last two coordinates $v^{(I I)} \equiv\left(v_{3}, v_{4}\right)$ correspond to the toric fiber direction. For each point $v^{F} \in \nabla_{2}$, the point $v=\left(0 ; v^{F}\right)$ lies in $\nabla .^{2}$ The primitive rays defining the base as a toric variety are those that are not integer multiples of another ray,

$$
\left\{v^{B}\right\}=\left\{v^{(I)}=\left(v_{1}, v_{2}\right) /\left(\operatorname{GCD}\left(v_{1}, v_{2}\right)\right), \exists v^{(I I)}: v=\left(v^{(I)}, v^{(I I)}\right) \in \nabla\right\} .
$$

The existence of the projection $\pi: v=\left(v_{1}, v_{2} ; v_{3}, v_{4}\right) \rightarrow\left(v_{1}, v_{2} ; 0,0\right)$ taking $\nabla_{2} \rightarrow 0$ is equivalent to the condition that there is a projection on the dual lattice $\rho: m=$ $\left(m_{1}, m_{2} ; m_{3}, m_{4}\right) \rightarrow\left(0,0 ; m_{3}, m_{4}\right)$ that maps $\Delta$ to the mirror fiber $\tilde{F}=\Delta_{2} \cdot{ }^{3}$ For each $m^{(I I)} \in \Delta_{2}$, the set of lattice points in $\Delta$ that map under $\rho$ to $m^{(I I)}$ can be thought of as monomials that are sections of a specific line bundle over $B$. Each ray of the form (2.5) satisfies the condition $v \cdot m \geq-1$, which implies $\left(v_{1}, v_{2}\right) \cdot\left(m_{1}, m_{2}\right) \geq-1-v^{(I I)} \cdot m^{(I I)}$.

\footnotetext{
${ }^{2}$ Note that $v^{(I I)}$ in the fiber direction may lie outside the fiber $\nabla_{2}$ for a general point $\left(v^{(I)} ; v^{(I I)}\right)$ in $\nabla$. In fact, as we discuss further later in the paper, this has to be the case for some lattice points in $\nabla$ when the dual polytope $\Delta$ is not a fibered polytope.

${ }^{3}$ This can be easily proven as follows: given the projection $\pi$, we know that the fiber $\nabla_{2}$ lies in the plane $(0,0 ; \cdot, \cdot)$. This implies that any $m=\left(m_{1}, m_{2} ; m_{3}, m_{4}\right) \equiv\left(m^{(I)} ; m^{(I I)}\right)$ satisfies $m^{(I I)} \cdot v^{F} \geq-1, \forall v^{F} \in \nabla_{2}$, which implies $m^{(I I)} \in \Delta_{2}$, i.e., the existence of the projection $\rho$ onto $\Delta_{2}$. To see the existence of the fiber given the projection $\rho$, every point in the form $v=\left(0,0 ; v^{F}\right)$, where $v^{F} \in \nabla_{2}$, satisfies $v \cdot m \geq-1, \forall m \in \Delta$ since $v^{F} \cdot m^{(I I)} \geq-1, \forall m^{(I I)} \in \Delta_{2}$; we therefore have $\nabla_{2} \subset \nabla$, and the projection taking $\nabla_{2} \rightarrow 0$ takes the form $\pi$.
} 
When $v^{B}=\left(v_{1}, v_{2}\right)$ is a primitive ray and corresponds to a toric curve $C$ in the base $B$, this means that $m^{(I)}=\left(m_{1}, m_{2}\right)$ is a section of a line bundle that can vanish to order $v^{(I I)} \cdot m^{(I I)}+1$ on $C$.

The simplest examples of the utility of these conditions can be seen in polytopes that have the "stacked" form described in $[15,16,28]$, where there is a fixed lattice point $v_{s} \in \nabla_{2}$ so that for every ray $v^{B}$ in the base there exists a ray of the form $\left(v^{B} ; v_{s}\right) \in \nabla$ for that particular lattice point $v_{s}$. In these cases, the monomials over $m^{(I I)}$ represent sections of the line bundle $\mathcal{O}\left(-n K_{B}\right)$, where $n=1+v^{(I I)} \cdot m^{(I I)}$ and $-K_{B}$ is the anti-canonical class of the base. In particular, when the fiber is $F_{10}=\mathbb{P}^{2,3,1}$, and $v_{s}=(-3,-2)$ the resulting "standard stacking" form gives monomials over the points in $\Delta_{2}$ that naturally describe the general (Tate) form of the Weierstrass model for an elliptic fibration, and can be described as sections of $\mathcal{O}\left(-n K_{B}\right)$, with $n=1,2,3,4,6$.

\section{$2.2 \quad$ Factorization of mirror symmetry}

From the preceding characterization of polytope fibrations, it clearly follows that under certain circumstances when the polytope $\nabla$ has a subpolytope $\nabla_{2}=F_{i}$ that gives a genus one fibration of the associated Calabi-Yau hypersurface $X$ the polytope $\Delta$ will also have a subpolytope $\Delta_{2}=\tilde{F}_{i}$ that gives a genus one fibration of the mirror Calabi-Yau $\tilde{X}$. This will occur whenever there is a coordinate system such that the point $\left(0,0 ; m^{(I I)}\right)$ is a point in $\Delta$ for all $m^{(I I)} \in \Delta_{2}$. A necessary and sufficient condition for this to occur is that there exist a coordinate system so that every lattice point in $\nabla$ can simultaneously be put in the form (2.5), with $v^{(I I)}=v^{F} \in \nabla_{2}$ (the values of $v^{F}$ need not be the same for different lattice points in $\nabla$ but they must all lie in $\nabla_{2}$ ), i.e. that there is a projection in the space $N$ onto the fiber polytope $\nabla_{2} \cdot{ }^{4}$

In any situation where these conditions hold, we have a mirror pair of Calabi-Yau manifolds ${ }^{5} X, \tilde{X}$, each of which is elliptically or genus one fibered. Furthermore in the toric presentation, the 2D toric fibers associated with the elliptic or genus one fibers of $X, \tilde{X}$ themselves have mirror hypersurface curves, with $F=F_{i}, \tilde{F}=\tilde{F}_{i}$. We refer to this situation as a "factorization" of mirror symmetry for elliptic Calabi-Yau manifolds.

\footnotetext{
${ }^{4}$ This condition was encountered in the context of K3 surfaces and heterotic F-theory duality in several earlier papers; mirror K3 fibrations were described in terms of slices and projections in [8, 17], and described in terms of symplectic cuts in [19], motivated by some examples found by Candelas. This type of construction has been further used in studying mirror symmetry of $G_{2}$ manifolds [29]. The strong prevalence of mirror symmetric pairs of K3-fibered CY3s observed in [8] is closely related to the prevalence of mirror symmetric pairs of elliptically-fibered CY3s studied here; indeed, many of the examples we consider here are also K3 fibered.

${ }^{5}$ The polytopes in the KS database [6] are associated with the monomial polytopes $\Delta$. Given a mirror pair of fibered polytopes $\nabla, \Delta$, where $\nabla$ is the "fan polytope" associated with the fibration $X$ with the Hodge numbers $\left(h^{1,1}(X), h^{2,1}(X)\right)$, it is the $\Delta$ polytope associated with the data M:\# lattice points, \# vertices (of $\Delta$ ) N:\# lattice points, \# vertices (of $\nabla) \mathrm{H}: h^{1,1}(X), h^{2,1}(X)$ that is listed on the website. Then $\Delta$ is the fan polytope associated with the fibration $\tilde{X}$ with the Hodge numbers $\left(h^{1,1}(\tilde{X})=h^{2,1}(X)\right.$, $\left.h^{2,1}(\tilde{X})=h^{1,1}(X)\right)$ While the lattice points in the fan polytope $\nabla$ associated with the fibration $X$ are denoted by $v$, when $\Delta$ is viewed as the fan polytope associated with the fibration $\tilde{X}$, we sometimes denote the lattice points in $\Delta$ by $w$, while we use the same symbol $m$ to denote the lattice points in either $\Delta$ or $\nabla$ in the cases when they are used as the monomial polytope.
} 
This kind of factorization is really in some sense a semi-factorization. In particular, the relationship between the base $B$ of the elliptic fibration of $X$ and the base $\tilde{B}$ of the mirror fibration depends upon the "twist" of the fibration of $X$ encoded in the specific way in which the rays of the base lie over the fiber in (2.5). In general, this relationship can be rather complex, though it can always be determined from the condition described in section 2.1 that implies that each primitive base ray in the mirror is associated with a section $m^{(I)}$ of a line bundle whose degree of vanishing on the associated curve is constrained by the set of inner products $v^{(I I)} \cdot m^{(I I)}$.

In many cases, the structure is particularly simple and the base $\tilde{B}$ of the mirror elliptic fibration can be associated with a line bundle $\mathcal{O}\left(-n K_{B}\right)$ for a fixed $n$, so that $\tilde{B}$ can be read off in a simple way from $B$. In particular, this occurs when all the rays of the base are stacked over a particular point $v_{s} \in \nabla_{2}$ in the fiber, and there are no rays in $\nabla$ (associated with "tops" [30]) representing rays in the base over other fiber points that impose extra constraints on the points in the mirror polytope. In this case, the monomials in the dual polytope $\tilde{B}$ can be associated with sections of line bundles $\mathcal{O}\left(-n K_{B}\right)$, with $n=1+v_{s} \cdot m^{(I I)}$, and the base $\tilde{B}$ can be associated with a polytope built from the primitive rays in the set of points in the $2 \mathrm{D}$ polytope associated with $-n K_{B}$ with the largest value of $n$ realized from the points $m^{(I I)} \in \Delta_{2}$ :

$$
\left\{w_{i}^{\tilde{B}}\right\}=\left\{w=\left(w_{1}, w_{2}\right) \mid \operatorname{GCD}\left(w_{1}, w_{2}\right)=1, w \cdot v^{B} \geq-n_{v_{s}} \forall v^{B} \in \Sigma_{B}\right\}
$$

where $\Sigma_{B}$ is the toric fan for $B$ and $n_{v_{s}}=\max \left(\left\{\left(1+v_{s} \cdot m^{(I I)}\right) \mid m^{(I I)} \in \Delta_{2}\right\}\right)$. In appendix A we illustrate for each $v_{s} \in \nabla_{2}$ the maximum value of $1+v_{s} \cdot m^{(I I)}$. Note that unlike in (2.6), where we are dealing with projections of $4 \mathrm{D}$ rays, when an integer multiple $k w$ of a primitive $2 \mathrm{D}$ vector $w$ satisfies $k w \cdot v^{B} \geq-n$ then the vector $w$ also satisfies this condition, so we can construct all primitive vectors by simply taking those with unit GCD on the coordinates. The simplest cases in which (2.7) applies is for the "standard stacking" $F_{10}$ fiber constructions associated with the generic Tate form for an elliptic fibration over a toric base, as discussed at the end of section 2.1, in which case $n_{v_{s}}=6$. We describe a number of examples of this type in section 3. When there is a further tuning of the monomials in $\Delta$, associated with a nontrivial "top" in $\nabla$, the construction of the mirror base $\tilde{B}$ is similar but depends on the tuning, as we discuss in more detail in section 4 .

All of the analysis just outlined is equally relevant taking mirror symmetry the other way. Starting with $\Delta$, the base $B$ can similarly in the complementary cases, such as when $\Delta$ is a standard stacking associated with a generic elliptic fibration over $\tilde{B}$, be calculated using (2.7) from $\mathcal{O}\left(-n_{w_{s}} K_{\tilde{B}}\right)$, where $w_{s} \in \Delta_{2}$ is the stacking point of the stacked polytope $\Delta$, and $n_{w_{s}}=\max \left(\left\{\left(1+w_{s} \cdot m^{(I I)}\right) \mid m^{(I I)} \in \nabla_{2}\right\}\right)$, and the same kind of generalization is used when there is a tuning of the monomials in $\nabla$. In the remainder of the paper we consider primarily examples of this type, where there is a clear factorization of the mirror symmetry that allows a ready identification of both the fiber and the base of the mirror fibered Calabi-Yau variety. We leave a further analysis of more general cases for future work. 


\section{Generic Calabi-Yau elliptic fibrations over toric base surfaces}

In this section we consider the simplest and perhaps clearest class of examples of the factorization of mirror symmetry described above: Calabi-Yau threefolds $X$ that are generic CY elliptic fibrations over a toric base surface $B$. The closely related class of threefolds resulting from tuned Tate-form Weierstrass models over a toric base provide a larger class of examples where the mirror symmetry factorization can be understood easily; examples of this broader class are given in section 3.4, section 4.1, and section 4.2.

\subsection{General case}

It was shown in [31] that there are 61,539 toric bases that support an elliptic Calabi-Yau threefold with a smooth resolution. ${ }^{6}$ The Hodge numbers of the generic elliptic fibrations over all these bases were analyzed in [7]. It was shown in [15] that for each toric base $B$, a reflexive $4 \mathrm{D}$ polytope in the Kreuzer-Skarke database can be constructed by starting with a "standard stacking" polytope defined by the convex hull of the set of points of the form (2.5), where the first two coordinates are taken across all toric rays $v^{B}$ in the fan of $B$, and the last two coordinates $v_{s}=(-3,-2)$ correspond to a vertex of the fiber $F=F_{10}=\mathbb{P}^{2,3,1}$, and then taking the "dual of the dual" of the resulting polytope. In the simplest cases, where $B$ only has curves of self-intersection $-n$ where $n \mid 12$, the initial polytope is already reflexive. In all these cases, there is a corresponding reflexive 4D polytope in the Kreuzer-Skarke database that has an explicit $F_{10}$ fiber.

For each of these generic elliptic fibrations over a toric base, the dual polytope $\Delta$ associated with the mirror Calabi-Yau threefold $\tilde{X}$ contains lattice points that can be interpreted as sections of line bundles $\mathcal{O}\left(-n K_{B}\right)$ with $n=1,2,3,4,6$. These correspond to the coefficient polynomials in the "Tate form" of a Weierstrass model ${ }^{7}$

$$
y^{2}+a_{1} y x+a_{3} y=x^{3}+a_{2} x^{2}+a_{4} x+a_{6},
$$

where $a_{n}$ is a section of $\mathcal{O}\left(-n K_{B}\right)$. Since the origin is contained in each of the $2 \mathrm{D}$ sets of points over each $m^{(I I)} \in \Delta_{2}$, for all these models the dual polytope $\Delta$ has a subpolytope $\tilde{F}=\Delta_{2}$ lying on the plane $m_{1}=m_{2}=0$, so the resulting mirror $\tilde{X}$ also has an elliptic fiber given by an anti-canonical curve in the toric fiber $\tilde{F} \cong F=F_{10}$. These correspond to one of the simplest classes of elliptic toric hypersurface Calabi-Yau threefolds with a simple and manifest factorization of mirror symmetry. In these cases, the dual polytope has a base that is described as a toric variety by the set of primitive rays associated with the monomials in $\mathcal{O}\left(-6 K_{B}\right)$, which lie over the point $m^{(I I)}=(-1,-1) \in \Delta_{2}$. The Hodge

\footnotetext{
${ }^{6}$ Note that these bases include those with curves of self-intersection $-9,-10,-11$. Such bases can be blown up at non-toric points to achieve a smooth flat fibration Calabi-Yau resolution; Calabi-Yau threefolds can also be realized as non-flat fibrations over these bases. This technicality is handled automatically through the resolution process for the toric hypersurface Calabi-Yau threefolds in the Kreuzer-Skarke database [15] (see also examples in table 2 and an example in section 4.3.)

${ }^{7}$ The term "Tate form" is used often by physicists for this general form of the Weierstrass model because of its use in the context of F-theory in the Tate algorithm for constructing models with a particular desired gauge group.
} 


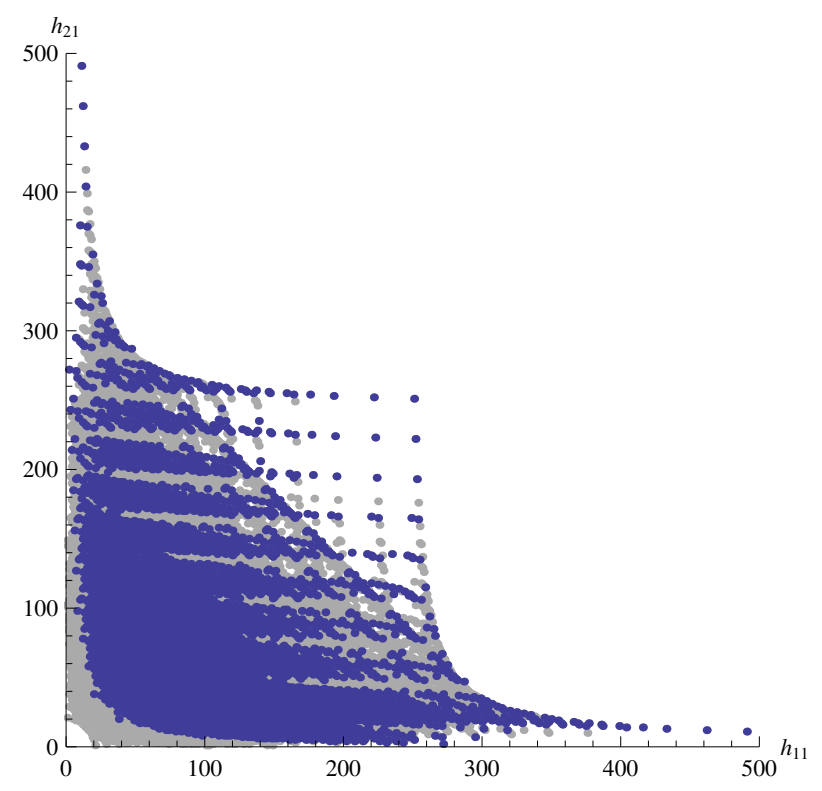

Figure 1. The Hodge numbers (blue points) of Calabi-Yau threefolds $X$ formed as generic elliptic fibrations over toric base surfaces. All these examples have a particularly simple form of factorized mirror symmetry in which $X$ and $\tilde{X}$ both have elliptic fibers described as anti-canonical curves in the toric fiber $F_{10}=\mathbb{P}^{2,3,1}$ and the mirror base $\tilde{B}$ is constructed as a toric variety from the monomials in the line bundle $\mathcal{O}\left(-6 K_{B}\right)$, where $B$ is the base of the elliptic fibration of $X$. Gray points are Hodge numbers in the full KS database.

numbers of the generic elliptic fibrations over toric bases are plotted in figure 1 [7]; these include many of the largest Hodge number pairs in the KS database. ${ }^{8}$

In the remainder of this section we describe some examples of these factorized mirror pairs explicitly. From this construction, it is clear that similarly, an arbitrary Tate tuning of the generic elliptic fibration over a toric base $B$, which is realized by a reduction in the set of monomials in $\Delta$ and an increase in the set of rays in $\nabla$ (often described in the language of "tops" [30]), as described in more detail in [15], will also lead to a mirror pair of Calabi-Yau threefolds that are both elliptically fibered with the self-dual toric 2D fiber type $F_{10}$. In general, tuning the fibration $X$ will reduce the size of the polytope associated with the mirror base $\tilde{B}$, which will then be described by a toric fan that contains as rays only a subset of the primitive rays in $-6 K_{B} \cdot{ }^{9}$ We describe examples of such tunings in sections 4.1-4.3.

\footnotetext{
${ }^{8}$ The simplest subset of these cases, where both sides of the mirror symmetry are generic elliptic fibrations without tuning, and some of the patterns appearing in these cases, were noted in the context of an earlier project with Braun and Wang [32].

${ }^{9}$ Note that in some cases when all monomials over some points $m^{(I I)} \in \Delta_{2}$ are set to vanish, this furthermore will correspond to reducing the size of $\Delta_{2}$, with a corresponding increase in the size of $\nabla_{2}$; for example, setting $a_{6}=0$ in the Tate model changes the dual fiber to $\tilde{F}_{13}$, so that the fiber of $\nabla$ acquires two additional points and becomes $F_{13}$.
} 


\subsection{Example: generic elliptic fibration over $\mathbb{P}^{2}$ (Hodge numbers $(2,272)$ )}

As a simple first case, we consider the case of the base $B=\mathbb{P}^{2}$, with a generic elliptic fiber given by an anti-canonical curve in the toric fiber space $\mathbb{P}^{2,3,1}$ (i.e. the toric fiber $F_{10}$ ). In this case the polytope $\nabla$ has vertices

$$
\left\{v_{i}\right\}=\{(0,0 ; 1,0),(0,0 ; 0,1),(1,0 ;-3,-2),(0,1 ;-3,-2),(-1,-1 ;-3,-2)\} .
$$

Here the fiber is given by the slice with vertices $(0,0 ; 1,0),(0,0 ; 0,1),(0,0 ;-3,-2)$, and the projection onto the base projects the last two coordinates to 0 . Since $B$ contains no curves of self-intersection $-n$ with $n$ not a divisor of $12, \nabla$ is immediately reflexive. The dual polytope $\Delta$ is easily seen to have vertices (see e.g. [15] for explicit analysis)

$$
\left\{m_{i}\right\}=\{(0,0 ;-1,2),(0,0 ; 1,-1),(-6,12 ;-1,-1),(12,-6 ;-1,-1),(-6,-6 ;-1,-1)\} .
$$

Under a coordinate transformation on the last two coordinates this takes the form

$$
\left\{w_{i}\right\}=\{(0,0 ; 1,0),(0,0 ; 0,1),(-6,12 ;-3,-2),(12,-6 ;-3,-2),(-6,-6 ;-3,-2)\} .
$$

This dual polytope $\Delta$ is again fibered by an $F_{10}$ fiber in the $(0,0 ; \cdot, \cdot)$ plane, and the projection onto the base gives a base $\tilde{B}$ that has a toric description using the primitive rays in the $2 \mathrm{D}$ polytope with vertices $(-6,12),(12,-6),(-6,-6)$ (See figure 2$)$. The dual base polytope consists of the points in $\Delta$ that can be associated with sections of the line bundle $-6 K_{B}$. The mirror polytopes $\nabla, \Delta$ both appear in the Kreuzer-Skarke database, and give rise to elliptic toric hypersurface Calabi-Yau threefolds with hodge numbers (2, $272)$ and $(272,2)$ respectively (see footnote 5$)$.

We can characterize the mirror base $\tilde{B}$ as a toric variety by the sequence of selfintersections of the toric rays calculated by equation (2.7)

$$
\tilde{B} \rightarrow[[-11 / /-12 / /-12 / /-11 / /-12 / /-12 / /-11 / /-12 / /-12 / /]]
$$

where the notation // denotes the sequence of self-intersections

$$
/ /=-1,-2,-2,-3,-1,-5,-1,-3,-2,-2,-1 .
$$

This sequence of self-intersections is a familiar sequence that connects -12 curves that support $E_{8}$ (Kodaira type $I I^{*}$ ) singularities in the elliptic fibration (see e.g. [33, 34]). This sequence of self-intersections characterizes a face of the base at distance 6 from the origin; similar structure for faces at different distances from the origin are described in appendix B.

Using methodology motivated by F-theory we can compute the Hodge numbers of the generic elliptic fibrations over $B, \tilde{B}$ directly from the geometry of the bases [7, 35, 36]. For $B$, all curves have self-intersection above -3 , so there is no non-Higgsable gauge group. From the Shioda-Tate-Wazir formula we have

$$
h^{1,1}(X)=h^{1,1}(B)+1=2 .
$$

From the gravitational anomaly condition, we have

$$
h^{2,1}(X)=273-29\left(h^{1,1}(B)-1\right)-1=272 .
$$




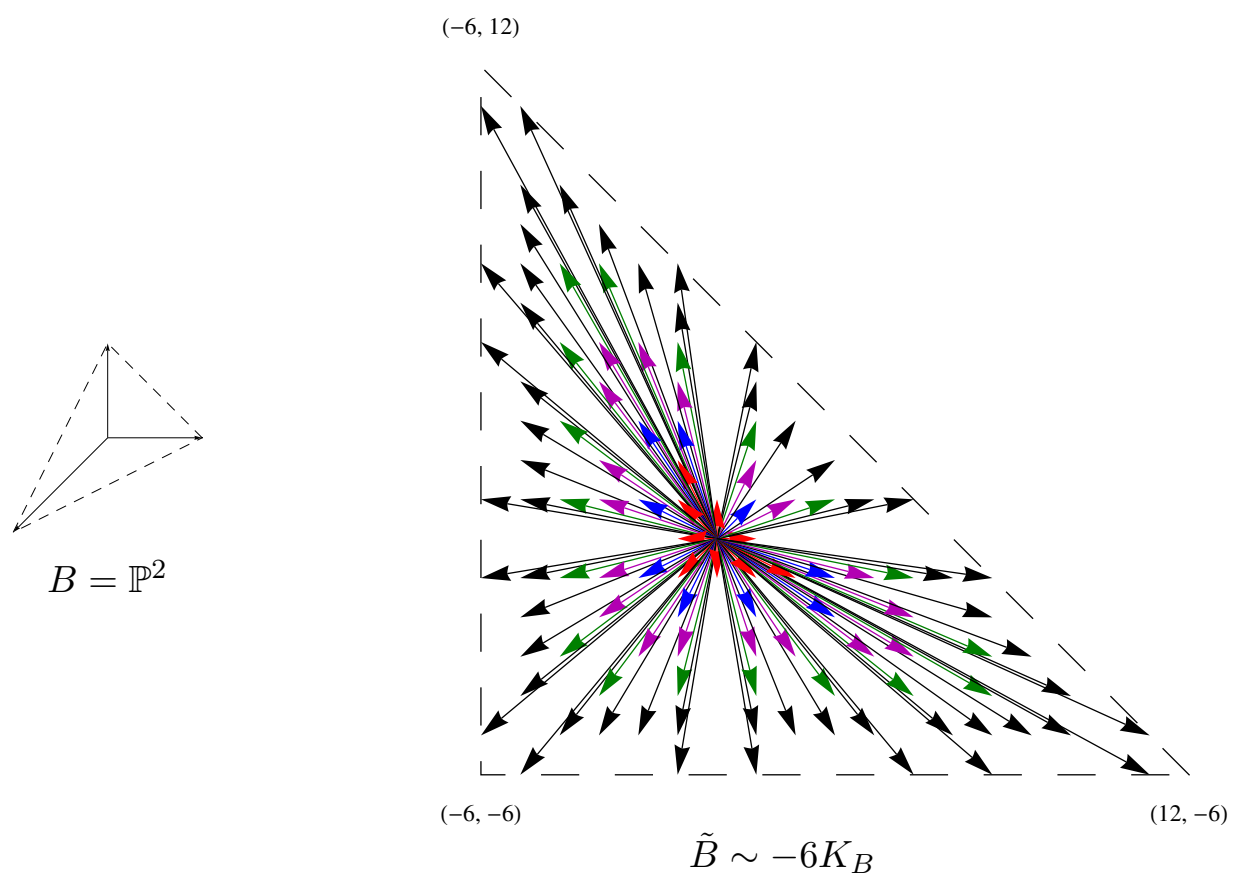

Figure 2. The base $B=\mathbb{P}^{2}$ and the base $\tilde{B}$ of the mirror of the generic elliptic fibration over $B$, shown as toric varieties. Rays that are red, blue, purple, green correspond to curves in the toric base $\tilde{B}$ that carry non-Higgsable $E_{8}, F_{4}, G_{2}$, and $\mathrm{SU}(2)$ gauge groups respectively.

For the mirror the computation is a bit more complicated. On each -11 curve there is a single $(4,6)$ point that must be blown up so that the total space has a smooth Calabi-Yau resolution [33]. Before these blowups the number of toric curves is 108, so $h^{1,1}(\tilde{B})=108-2+3=109$. The non-Higgsable gauge group from the curves of negative self-intersection below -1 is $G=E_{8}^{9} \times F_{4}^{9} \times\left(G_{2} \times \mathrm{SU}(2)\right)^{18}$, with rank 162 . We then have

$$
h^{1,1}(\tilde{X})=h^{1,1}(\tilde{B})+\operatorname{rank} G+1=109+162+1=272 .
$$

On the other hand, each $G_{2} \times \mathrm{SU}(2)$ non-Higgsable factor is associated with 8 charged matter hypermultiplets, so we have

$$
\begin{aligned}
h^{2,1}(\tilde{X}) & =273-29\left(h^{1,1}(\tilde{B})-1\right)+\operatorname{dim} G-m_{\mathrm{NH}}-1 \\
& =273-29(108)+9(248+52+34)-144-1=2 .
\end{aligned}
$$

\subsection{Example: self-mirror Calabi-Yau threefold with Hodge numbers (251, 251)}

We now consider the self-mirror Calabi-Yau threefold with Hodge numbers $(251,251)$ at the central peak of the "Hodge shield". This polytope can be put into a coordinate system where the vertices are

$$
\left\{v_{i}\right\}=\{(0,0 ; 1,0),(0,0 ; 0,1),(-1,6 ;-3,-2),(0,-1 ;-3,-2),(42,6 ;-3,-2)\} .
$$

This polytope is self-dual, $\nabla=\Delta$, up to a coordinate transformation, and is clearly a $\mathbb{P}^{2,3,1}$ fibration over the base $B$ with vertices $(-1,6),(0,-1),(42,6)$. Since the inner product 


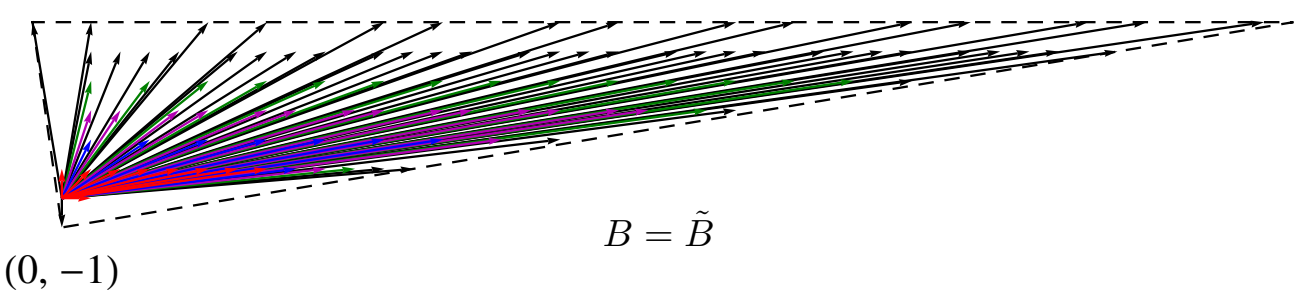

Figure 3. The base $B=\tilde{B}$ over which the generic elliptic fibration is the self-mirror Calabi-Yau threefold with Hodge numbers $(251,251)$. Note that the inner product between each pair of vertices is -6 , so that $B=\tilde{B} \sim-6 K_{B}$. Rays that are red, blue, purple, green correspond to curves in the toric base $\tilde{B}$ that carry non-Higgsable $E_{8}, F_{4}, G_{2}$, and $\mathrm{SU}(2)$ gauge groups respectively.

between each pair of these vertices is -6 , the procedure of constructing the mirror base $\tilde{B}$ from monomials in $\mathcal{O}\left(-6 K_{B}\right)$ gives $B=\tilde{B}$. The primitive rays in this base (figure 3 ) give a toric surface with a sequence of self-intersections

$$
[[0,6,-12 / /-11 / /-12 / /-12 / /-12 / /-12 / /-12 / /-12 / /-12]] .
$$

Again using the relationship between geometry and F-theory physics we can compute the Hodge numbers directly from the geometry of the base. There are 99 toric curves in the base, with one -11 curve that must be blown up, so $h^{1,1}(B)=99-2+1=98$. The non-Higgsable gauge group is $G=E_{8}^{9} \times F_{4}^{8} \times\left(G_{2} \times \mathrm{SU}(2)\right)^{16}$, with rank 152, so

$$
h^{1,1}(X)=h^{1,1}(B)+\operatorname{rk} G+1=251 .
$$

Similarly,

$$
h^{2,1}(\tilde{X})=273-29\left(h^{1,1}(B)-1\right)+\operatorname{dim} G-m_{\mathrm{NH}}-1=251 .
$$

\subsection{Example: generic elliptic fibration over $\mathbb{F}_{n}$ (Hodge numbers $(3,243), \ldots$, $(11,491)$}

As further examples we consider the generic elliptic fibrations over the Hirzebruch surfaces $B=\mathbb{F}_{n}$. In each case the mirror Calabi-Yau is elliptic over a base constructed from $-6 K_{B}$, though in some cases the mirror is not a generic elliptic fibration but has some tuning. We describe several specific cases in detail and summarize the complete set for all $n=0, \ldots, 12$ in table 1 and table 2. The Hirzebruch surface $\mathbb{F}_{n}$ can be described by the toric rays $(0,1),(1,0),(0,-1),(-1,-n)$. For $n=0,1$ these are the vertices of the associated 2D polytope, while for $n>1(0,-1)$ is not a vertex and the other three are. The dual base $\tilde{B} \sim-6 K_{B}$ is thus characterized by the toric variety with a fan given by all primitive rays in the polytope defined by the vertices

$$
\{(-6,-6),(-6,12 / n),(6(n+1),-6)\},
$$

when $n \geq 2,\{(-6,-6),(-6,12),(6,0),(6,-6))\}$ for $n=1$, and $\{(-6,-6),(-6,6),(6,6)$, $(6,-6)\}$ for $n=0$. 


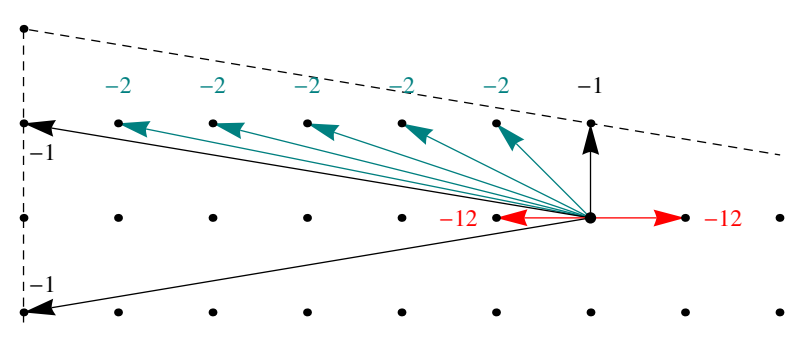

Figure 4. Part of the ray structure of the toric base $\tilde{B}$ for the mirror $\tilde{X}$ of the generic elliptic fibration over the Hirzebruch surface $\mathbb{F}_{6}$.

When $n \mid 12, n>1$, the vertices of the polytope (3.15) containing the dual base are integral; from this it follows that the polytope $\nabla$ is reflexive, since $\Delta$ has the form of (3.3), where the last set of vertices are given by $\left(m^{(I)} ;-1,-1\right)$, with $m^{(I)}$ vertices of the polytope (3.15). The polytope $\nabla$ has vertices [15]

$$
\left\{v_{i}\right\}=\{(0,0 ; 1,0),(0,0 ; 0,1),(1,0 ;-3,-2),(0,1 ;-3,-2),(-1,-n ;-3,-2)\} .
$$

As in the preceding examples we can read off the sequence of self-intersections of the toric rays associated with primitive rays in (3.15). We give a few explicit examples.

$\mathbb{F}_{12}$. The polytope $\nabla$ is reflexive, and the Hodge numbers of the associated Calabi-Yau threefold are $(11,491)$; this is the largest possible value of $h^{2,1}(X)$ for any elliptic CalabiYau threefold [7]. In this case the vertex $(-6,1)$ of $\tilde{B}$ is primitive and corresponds to a curve of self-intersection 0 , and the vertices $(-6,-6)$ and $(78,-6)$ each go to primitive rays $(-1,-1)$ and $(13,-1)$ associated with curves of self-intersection 11 . The sequence of self-intersections of the toric rays for $\tilde{B}$ is then

$$
\left[\left[-12 / /-11 / /(-12 / /)^{13}-11 / /-12,0\right]\right]
$$

Blowing up the base at two points on the -11 curves we can confirm that the Hodge numbers of the generic elliptic fibration, corresponding to the polytope $\Delta$, are $(491,11)$ as expected.

$\mathbb{F}_{\text {6. }}$ In this case again $\nabla$ defined through (3.16) is immediately reflexive. The Hodge numbers for the associated Calabi-Yau threefold are $(9,321)$, which can be immediately determined from the non-Higgsable $E_{6}$ gauge group over the -6 curve in $B$. In this case, however, the vertex $(-6,2)$ is not primitive. The top part of the toric diagram for $\tilde{B}$ is shown in figure 4 , and contains the sequence of curves of self-intersection $-1,-2,-2,-2,-2,-2,-1$. Unlike in the case of $\mathbb{F}_{12}$ the mirror polytope $\Delta$ is not a generic elliptic fibration over $\tilde{B}$. And indeed, the generic elliptic fibration over $\tilde{B}$ has Hodge numbers $(317,17)$ rather than the values of $(321,9)$ expected from mirror symmetry. This can be understood from the fact that the vertex $(-6,2)$ is not present in $\tilde{B}$, so the monomials in $-6 K_{\tilde{B}}$ are not simply the points in $B=\mathbb{F}_{6}$ but also include the lattice points $(0,-k), k \in\{4,5,6\}$. In the polytope $\nabla$ these monomials are all set to vanish. Computing the resulting gauge group structure on $\tilde{B}$ we find that there is a gauge group $\mathrm{SU}(2) \times G_{2} \times \mathrm{SU}(2)$ tuned on the middle 
sequence of three -2 curves. This gives an additional rank contribution of 4 to $h^{1,1}(\tilde{X})$ from the gauge group and there are contributions to $h^{2,1}(\tilde{X})$ of +20 from the dimension of the tuned gauge group, and -28 from the charged matter fields, ${ }^{10}$ so that the correct Hodge numbers are found for the tuned Calabi-Yau

$$
(321,9)=(317,17)+(4,-8) .
$$

When $n$ does not divide 12 , there are additional vertices of $\nabla$ that must be included to attain a reflexive polytope from the simple stacking of $\mathbb{F}_{n}$.

$\mathbb{F}_{\mathbf{5}}$. For example, when $n=5,(0,-3,-3,-2)$ is an additional vertex that must be included with the set in (3.16) for $\nabla$ to be reflexive, ${ }^{11}$ so the projection of the whole $\nabla$ polytope to the base plane is instead the polytope defined by the vertices

$$
\{(1,0),(0,1),(-1,-5),(0,-3)\},
$$

and the convex hull of the toric fan of $B$ lies within this polytope. On the dual side, $\tilde{X}$ is a generic elliptic fibration with Hodge numbers $(295,7)$. The polytope defined by all monomials in $\mathcal{O}\left(-6 K_{B}\right)$ (defined using the rays in $B$ from equation (3.19)) has the set of vertices

$$
\{(-6,2),(-4,2),(-6,-6),(36,-6))\} \text {. }
$$

This is the projected polytope of the whole $\Delta$ to the base. The primitive rays in this projected polytope define the base $\tilde{B}$, which is characterized by the self-intersection sequence

$$
\left[\left[-12 / /-11 / /(-12 / /)^{6}-11 / /-12,-1,-2,-2,-3,-1,-3,-2,-2,-1\right]\right],
$$

and the vertices of the convex hull of the toric fan of $\tilde{B}$ are

$$
\{(-5,2),(-6,1),(1,1),(-6,-5),(-5,-6),(31,-5),(35,-6)\}
$$

which is contained in the projected polytope. In this case the mirror $\Delta$ is again a generic (non-tuned) elliptic fibration over the mirror base $\tilde{B}$, and the Hodge numbers can be computed directly from the non-Higgsable clusters on the base with intersections (3.21).

We list the results of the remaining $\mathbb{F}_{n}$ cases in table 1 for $0 \leq n \leq 8, n=12 .{ }^{12}$ For $n=9,10,11$, additional blowups at points in the base that may be toric or non-toric are required to support a flat elliptic fibration, and there are various ways to resolve these bases (see table 15 in [15]). We find that the corresponding mirror fibrations are generic models over different dual bases $\tilde{B}$ for different resolutions. The results are listed in table 2 .

\footnotetext{
${ }^{10}$ Note that the correct counting of $h^{2}, 1$ considers only the matter fields charged under the Cartan subalgebra. The counting in this case is equivalent to that of the rank preserving tuning of $\mathrm{SU}(2) \times$ $\mathrm{SU}(3) \times \mathrm{SU}(2)$, where the $\mathrm{SU}(3)$ has 6 hypermultiplets charged in the fundamental (3) representation, 4 of which are in bifundamentals with the $\mathrm{SU}(2)$ factors. In this case the $\mathrm{SU}(3)$ fundamentals combine in pairs into $G_{2}$ fundamentals, and the $G_{2}$ has one additional fundamental (7), which contributes another 6 hypermultiplets charged under the Cartan, canceling the difference in dimension between $\mathrm{SU}(2)$ and $G_{2}$. This rank-preserving tuning between $\mathrm{SU}(2)$ and $G_{2}$ connects two phases of the same Calabi-Yau geometry.

${ }^{11}$ Additional vertices from tops for all generic fibrations over Hirzebruch surfaces can be looked up in table 11 in [15].

${ }^{12}$ Thanks to Yinan Wang for a computation showing that tunings were missing in the $\mathbb{F}_{3}, \mathbb{F}_{4}$ examples in the original version of the paper.
} 


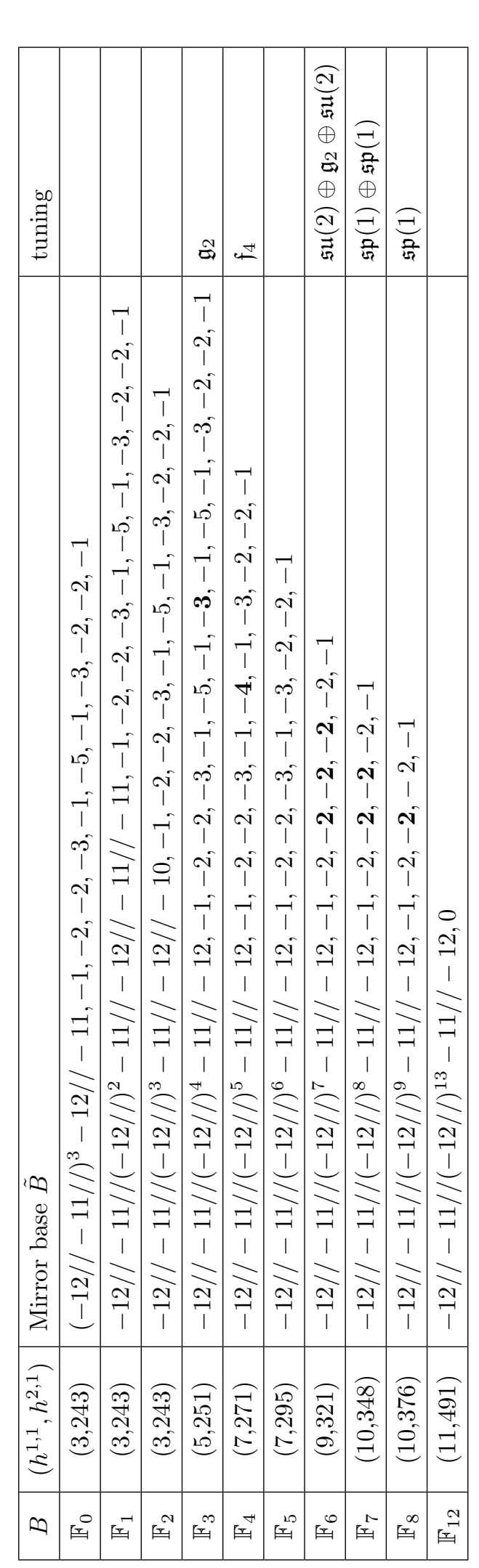

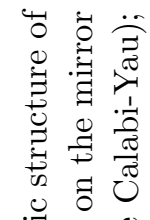

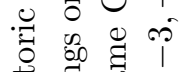

寻寻要

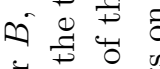

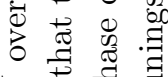

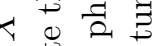

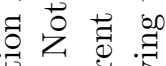

苛响事要

$\div \approx$

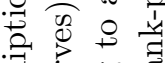

$\exists$

渮

वै क्ष

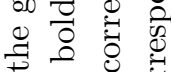

㟧

용용

छ्छ छ

$\exists \Xi \mathbb{D}_{0}$

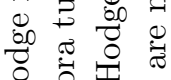

要

$\pm 0$

II

$\therefore \quad$

के สี

VI

Vl疒若苛

$\approx=0$

II

$\infty \exists \exists$

纯

\% .1 5 क

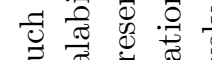

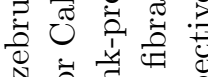

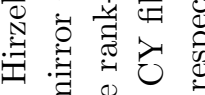

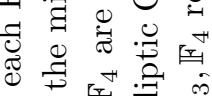

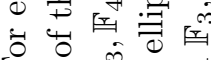

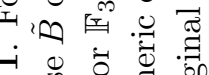

0.

जी 


\begin{tabular}{|c|c|c|c|}
\hline$B$ & $\left(h^{1,1}, h^{2,1}\right)$ & Resolved $B$ & Mirror base $\tilde{B}$ \\
\hline \multirow{6}{*}{$\mathbb{F}_{9}$} & \multirow{6}{*}{$(14,404)$} & & $-12 / /-11 / /(-12 / /)^{10}-11 / /-9,0$ \\
\hline & & $(*)$ & $-12 / /-11 / /(-12 / /)^{10}-11 / /-12,-1,-2,-2,-1$ \\
\hline & & & $-11 / /-11 / /(-12 / /)^{10}-11 / /-10,0$ \\
\hline & & & $-12 / /-11 / /(-12 / /)^{10}-11 / /-10,-1,-1$ \\
\hline & & & $-12 / /-11 / /(-12 / /)^{10}-11 / /-11,-1,-2,-1$ \\
\hline & & & $-11 / /-11 / /(-12 / /)^{10}-11 / /-11,-1,-1$ \\
\hline \multirow{4}{*}{$\mathbb{F}_{10}$} & \multirow{4}{*}{$(13,433)$} & \begin{tabular}{l|l}
-1 & \\
-12 & $(*)$ \\
\end{tabular} & $-12 / /-11 / /(-12 / /)^{11}-11 / /-10,0$ \\
\hline & & & $-12 / /-11 / /(-12 / /)^{11}-11 / /-12,-1,-2,-1$ \\
\hline & & $-\frac{12}{-12}$ & $-11 / /-11 / /(-12 / /)^{11}-11 / /-11,0$ \\
\hline & & -12 & $-12 / /-11 / /(-12 / /)^{11}-11 / /-11,-1,-1$ \\
\hline \multirow{2}{*}{$\mathbb{F}_{11}$} & \multirow{2}{*}{$(12,462)$} & -12 & $-12 / /-11 / /(-12 / /)^{12}-11 / /-11,0$ \\
\hline & & \begin{tabular}{|l|l}
-1 & $*$ \\
-12
\end{tabular} & $-12 / /-11 / /(-12 / /)^{12}-11 / /-12,-1,-1$ \\
\hline
\end{tabular}

Table 2. For each Hirzebruch base $B=\mathbb{F}_{n}, n=9,10,11$ the Hodge numbers of the generic elliptic fibration $X$ over resolved $B$, the toric structure of the resolved base and the base $\tilde{B}$ of the mirror Calabi-Yau threefold $\tilde{X}$. The bases marked with $\left(^{*}\right)$ are resolved at generic non-toric points, and correspond to the naive stacking models with the given Hirzebruch base, while the other bases are resolved at combinations of toric and non-toric points. 


\section{Some further examples}

In this section we consider some further examples that go beyond generic CY elliptic fibrations over toric bases. We first consider some cases of Tate tunings of the generic elliptic fibrations. As discussed in more detail in [15], a Tate tuning over a toric base generally gives a reflexive $4 \mathrm{D}$ polytope with a standard stacking form and the $F_{10}$ fiber, where the Tate tuning corresponds to reducing the monomials in $\Delta$. There is a natural correspondence between these Tate tunings and tops involving additional lattice points in $\nabla$. In general, for a Tate tuning where $a_{1}, \ldots, a_{6}$ vanish to orders $\left[a_{n}\right]_{i}$ on the toric divisor associated with the ray $v_{i}^{B}$, the vanishing of the monomials in $a_{n}$ corresponds to a removal of the points in $\Delta$ that lie over the point in $\Delta_{2}$ associated with sections of the line bundle $\mathcal{O}\left(-n K_{B}\right)$. For many Tate tunings, as for the generic elliptic fibration, the remaining points in the toric representation of $\mathcal{O}\left(-6 K_{B}\right)$ are a superset of the remaining points in the other $\mathcal{O}\left(-n K_{B}\right)$ 's. This is equivalent to the condition that the mirror polytope $\Delta$ has a standard stacking form so that all rays in the base have a preimage under the fiber projection of the form $(\cdot, \cdot ;-1,-1)$. In such situations, the set of rays of the dual base becomes

$$
\left\{w_{i}^{\tilde{B}}\right\}=V^{B}\left(a_{6}\right)
$$

where $^{13}$

$$
V^{B}\left(a_{n}\right)=\left\{w=\left(w_{1}, w_{2}\right) \mid \operatorname{GCD}\left(w_{1}, w_{2}\right)=1, w \cdot v_{i}^{B} \geq-n+\left[a_{n}\right]_{i} \forall v_{i}^{B} \in \Sigma_{B}\right\} .
$$

We describe explicit examples of this in section 4.1 and section 4.2. More generally, there are some Tate tunings (such as $\mathrm{SU}(6)$ ) that place such stronger constraints on $a_{6}$ than on other coefficients $a_{n}$. This can occur when $6-\left[a_{6}\right]_{i}<n-\left[a_{n}\right]_{i}$ for some $i$ and $n \leq 4$. In such cases, the mirror $\Delta$ is no longer a standard stacking but we can still give an explicit description of the base $\tilde{B}$ in terms of $B$ and the Tate tuning,

$$
\left\{w_{i}^{\tilde{B}}\right\}=\cup_{n \in\{1,2,3,4,6\}} V^{B}\left(a_{n}\right) .
$$

This is essentially a rewriting of (2.6) for the mirror polytope $\Delta$ when $\nabla$ is a standard stacking type polytope with the fiber $F_{10}$. We describe an explicit example of this in section 4.3. In terms of the monomial polytope $\Delta$, the set of points determined by equation (4.2) are given by

$$
V^{B}\left(a_{n}\right)=\left\{\left(m_{1}, m_{2}\right) / \operatorname{GCD}\left(m_{1}, m_{2}\right) \mid m=\left(m_{1}, m_{2}, m^{\tilde{F}}(n)\right) \in \Delta\right\},
$$

where $m^{\tilde{F}}(n)$ represents the two coordinates of the particular lattice point $m^{(I I)} \in \Delta_{2}$ that is associated with monomials in $a_{n}$. This formulation was used in some of the following explicit computations. In the coordinate system we adopt in this paper

$$
\begin{aligned}
& m^{\tilde{F}}(n=1,2,3,4,6)=\{(0,0),(-1,1),(0,-1),(-1,0),(-1,-1)\}, \\
& m^{F}(n=1,2,3,4,6)=\{(0,0),(-1,0),(-1,-1),(-2,-1),(-3,-2)\} .
\end{aligned}
$$

\footnotetext{
${ }^{13}$ Note that $V^{B}\left(a_{n}\right)$ can also be described simply as a set of primitive rays in $2 \mathrm{D}$ toric coordinates associated with the monomials in $a_{n}$, with the product $\Pi_{i} z_{i}$ of the toric variables $z_{i}$ associated with the rays of $\Sigma_{B}$ taken as the origin.
} 
For example, $V^{B}\left(a_{6}\right)=\left\{\left(m_{1}, m_{2}\right) / \operatorname{GCD}\left(m_{1}, m_{2}\right) \mid m \in \Delta\right.$ of the form $\left.\left(m_{1}, m_{2},-1,-1\right)\right\}$. In principle, we can use this same kind of analysis to describe other kinds of fibrations with general fiber types $F_{i}$, though details of the structure of tuned models will be different and depend on tops for the different fibers [25]. We leave a more complete analysis of the general situation to future work.

In section 4.4, we consider a case of a stacked fibration with another fiber type, in section 4.5 we illustrate a case of a fibered polytope where the mirror is not fibered, and in section 4.6 we give an example of the factorization of mirror symmetry for a CalabiYau fourfold constructed as a generic elliptic fibration over a toric threefold base. These examples simply illustrate some of the directions in which the framework developed here can be extended, and a more detailed analysis of these directions is left for the future.

\subsection{Tunings of generic fibrations (example: tuning an $\mathrm{SU}(2)$ on $\mathbb{P}^{2}$ )}

We consider tuning an $\mathrm{SU}(2)$ on one of the +1 -curves in the $\mathbb{P}^{2}$ base of the generic fibration model in section 3.2. The corresponding $\Delta$ polytope of the tuned model can be constructed by reducing the set of lattice points in the $\Delta$ polytope of the generic model (detailed examples of constructing tuned polytope models can be found in appendix A in [15]); this corresponds to the polytope in the case database with data: M:316 $7 \mathrm{~N}: 116 \mathrm{H}: 3,231$. The new Hodge numbers match with the prediction from F-theory physics of tuning the SU(2) on a +1 -curve $(2,272)+(1,-41)=(3,231)$. The standard stacking polytope $\nabla$ has vertices

$\left\{v_{i}\right\}=\{(0,1 ;-3,-2),(-1,-1 ;-3,-2),(1,0 ;-3,-2),(1,0 ;-2,-1),(0,0 ; 1,0),(0,0 ; 0,1)\}$.

Now there is a non-trivial top over the base divisor associated with the ray $(1,0)$ due to the $\mathrm{SU}(2)$ tuning over the divisor, which we can interpret in terms of a Tate tuning in $\Delta$. $\nabla$ has the usual $\left(0,0 ; v^{F}\right)$ form of $F_{10}$ fiber as the only $2 \mathrm{D}$ subpolytope $\nabla_{2}$. The dual polytope $\Delta$ has vertices

$$
\begin{aligned}
\left\{w_{i}\right\}= & \{(-4,-6 ;-1,-1),(-4,10 ;-1,-1),(-2,-2 ;-1,1),(-2,4 ;-1,1),(12,-6 ;-1,-1), \\
& (0,0 ;-1,2),(0,0 ; 1,-1)\} .
\end{aligned}
$$

The $\Delta$ polytope has multiple distinct fibrations, consisting of four $F_{10}$ fibers, three $F_{13}$ fibers, and one $F_{16}$ fiber. In particular, there is a fiber $\Delta_{2}$ dual to $\nabla_{2}$, which has lattice points also in the form $\left(0,0 ; w^{F}\right)$. This gives the mirror fibration of the $\Delta$ polytope $\tilde{X}$ when viewed as a fan polytope. ${ }^{14}$ We can calculate the dual base $\tilde{B}$ by equation (2.6) for the polytope $\Delta$, giving the self-intersection sequence

$$
\begin{aligned}
\tilde{B} \rightarrow & {[[-12 / /-12 / /-11 / /-12 / /-12,-1,-2,-2,-3,-1,-5,-1,-3,-2,} \\
& -1,-8,-1,-2,-3,-2,-1,-8,-1,-2,-3,-2,-1,-8,-1,-2,-3,-2, \\
& -1,-8,-1,-2,-3,-1,-5,-1,-3,-2,-2,-1]] .
\end{aligned}
$$

\footnotetext{
${ }^{14}$ Note that $\Delta$ does not project onto the other fibers (the projection strictly contains the other fibers); otherwise $\nabla$ would have had more than one fiber subpolytope.
} 
This is exactly the base over which the generic elliptic fibration has Hodge numbers $(231,3)$. Therefore, we know $\Delta$ is the fan polytope associated with this generic fibration model. This generic fibration is thus the mirror fibration to the tuned $\mathrm{SU}(2)$ fibration model.

Note that this base has a smaller toric diagram than the base described in (3.5). There are additional constraints on the rays $w$ in (2.7) in this case coming from base rays $v^{B}$ over points in the fiber $\nabla_{2}$ other than $v_{s}$. We can see how this works explicitly as an example of (4.1). Tuning an SU(2) gauge factor using a Tate tuning over the divisor in $\mathbb{P}^{2}$ associated with $v_{3}^{B}=(1,0)$ requires tuning the coefficients in (3.1) to vanish to orders $\left(\left[a_{1}\right],\left[a_{2}\right],\left[a_{3}\right],\left[a_{4}\right],\left[a_{6}\right]\right)=(0,0,1,1,2)$ on this divisor. Thus, the rays in $\tilde{B}$ are restricted to a subset of those in $\mathcal{O}\left(-6 K_{B}\right)$, which satisfy the additional condition $w \cdot v_{3}^{B} \geq-4$, so $w_{1} \geq-4$. This is already no stronger than the constraint on any of the other sections $\mathcal{O}\left(-n K_{B}\right), n \leq 4$, so we can use (4.1) and there are no other contributions from the more general formula (4.3). From figure 2 we see that the base $\tilde{B}$ is thence associated with the primitive rays in the polytope with vertices $(-4,10),(-4,-6),(12,-6)$. This matches perfectly with the projection from (4.7).

As $\Delta$ is also a generic elliptic fibration associated with a standard stacking polytope with stacking point $w_{s}=(-1,-1)$, we can calculate $B \sim \mathcal{O}\left(-6 K_{\tilde{B}}\right)$, and confirm that the monomials in $\mathcal{O}\left(-6 K_{\tilde{B}}\right)$ are all the lattice points of the form $(\cdot, \cdot,-3,-2)$ in the original polytope $\nabla$.

It is interesting to note that in this case while there is a tuning of the fibration on the $\nabla$ side, corresponding to a reduction in the size of $\tilde{B}$ on the $\Delta$ side, the mirror is still a generic elliptic fibration over the new base. We now consider a case where there are tunings on both sides.

\subsection{Tunings of generic fibrations over base $B$ and mirror base $\tilde{B}$}

We now consider an example where both the fibration and the mirror fibration are tuned models. There is only one polytope associated with a CY3 with Hodge numbers $(6,248)$, which is a standard stacking polytope. The polytope $\nabla$ has vertices

$$
\begin{aligned}
\left\{v_{i}\right\}= & \{(1,0 ;-3,-2),(0,1 ;-3,-2),(-1,-3 ;-3,-2),(0,-1 ;-1,0),(0,-2 ;-3,-2) \\
& (0,0 ; 1,0),(0,0 ; 0,1)\}
\end{aligned}
$$

The obvious $F_{10}$ fiber in the plane $(0,0 ; \cdot, \cdot)$ is the only fiber of $\nabla$. The associated CY3 is a Tate tuned model over the base $\mathbb{F}_{3}$ with $\mathfrak{s o}(7)$ gauge symmetry enhanced on the -3 -curve. The dual polytope $\Delta$ has vertices

$$
\begin{aligned}
\left\{w_{i}\right\}= & \{(24,-6 ;-1,-1),(0,2 ;-1,-1),(-6,-6 ;-1,-1),(-6,2 ;-1,-1),(-2,2 ;-1,0), \\
& (-4,2 ;-1,0)(0,0 ; 1,-1),(0,0 ;-1,2)\}
\end{aligned}
$$

The dual polytope $\Delta$ has three $F_{10}$ fibers and one $F_{13}$ fiber. The dual fiber with lattice points in the form $\{0,0 ; \cdot, \cdot\}$ gives the mirror fibration with Hodge numbers $(248,6) . \Delta$ is a standard stacking polytope with stacking point $w_{s}=(-1,-1)$ with respect to the fiber. The mirror base, by direct calculation (taking the rays of $\tilde{B}$ to be the primitive rays of all 
the projected $4 \mathrm{D}$ lattice points in $\Delta$ using $(2.6)$ on $\Delta)$ is

$$
\begin{aligned}
\tilde{B} \rightarrow & {[[-4,-1,-4,-1,-3,-2,-2,-1,} \\
& -12 / /-11 / /-12 / /-12 / /-12 / /-12 / /-11 / /-12,-1,-2,-2,-3,-1]] .
\end{aligned}
$$

The generic fibration over $\tilde{B}$ has Hodge numbers $(247,7)$, so this is a tuned model. By explicit analysis of the Weierstrass model over $\tilde{B}$ associated with the polytope $\Delta$, we know there are enhanced gauge symmetries $\mathfrak{s o}(9) \oplus \mathfrak{s p}(1) \oplus \mathfrak{s o}(9)$ on the first three curves $-4,-1,-4$. The Hodge number shifts calculated from F-theory physics (with shared matter representations carefully considered) are $(1,-1)$, which agrees with the Hodge numbers associated with $\Delta(247,7)+(1,-1)=(248,6)$.

Unlike the previous example, in this case we have tunings both on the original fan polytope $(\nabla)$ and on the mirror $(\Delta)$ side. Nonetheless, the tunings of gauge symmetries in $X$ and $\tilde{X}$ allow us to use equation (4.1) to calculate the bases in both cases: ${ }^{15}$

$$
\left\{w_{i}^{(\tilde{B})}\right\}=V^{B}\left(a_{6}\right) \text { and }\left\{v_{i}^{(B)}\right\}=V^{\tilde{B}}\left(\tilde{a}_{6}\right)
$$

where $a_{6}$ is associated with lattice points in $\Delta$ of the form $(\cdot, \cdot,-1,-1)$, and where $\tilde{a}_{6}$ is associated with lattice points in $\nabla$ of the form $(\cdot, \cdot,-3,-2)$ (cf. equations (4.5) and (4.6)). All the examples we have considered so far are cases in which equation (4.1) applies to the calculation of both $B$ and $\tilde{B}$. We now consider a case in which the more general formula (4.3) is required.

\subsection{Standard stacking $F_{10}$-fibered $\nabla$ vs. non-standard $F_{10}$-fibered $\Delta$}

We now consider tuning an $\mathrm{SU}(6)$ on one of the +1 -curves in the $\mathbb{P}^{2}$ base of the generic $\mathrm{CY}$ elliptic fibration model in section 3.2. The tuned model corresponds to the polytope M:207 $11 \mathrm{~N}: 158 \mathrm{H}: 7,154$ in the KS database. The new Hodge numbers match with the prediction from F-theory physics of tuning the SU(6) on a +1 -curve: $(2,272)+(5,-118)=(7,231)$. The standard stacking polytope $\nabla$ has vertices

$$
\begin{aligned}
\left\{v_{i}\right\}= & \{(0,0 ; 1,0),(0,1 ;-3,-2),(-1,-1 ;-3,-2),(1,0 ;-1,-1),(1,0 ;-3,-2),(0,0 ; 0,1), \\
& (1,0 ; 0,1),(1,0 ; 0,0)\} .
\end{aligned}
$$

The dual polytope $\Delta$ has vertices

$$
\begin{aligned}
\left\{w_{i}\right\}= & \{(-1,-4 ;-1,0),(-1,-2 ;-1,1),(-1,-1 ; 0,0),(-1,2 ; 0,0), \\
& (-1,3 ;-1,1),(-1,5 ;-1,0),(0,-6 ;-1,-1),(0,0 ;-1,2), \\
& (0,0 ; 1,-1),(0,6 ;-1,-1),(12,-6 ;-1,-1)\} .
\end{aligned}
$$

This is however not a standard stacking $\mathbb{P}^{2,3,1}$ polytope, which can be seen from the feature that there is more than one monomial in the coefficients of $x^{3}$ or $y^{2}$ in the Tate form [15]:

\footnotetext{
${ }^{15}$ We can safely use equation (4.1) without going to the general formula (4.3) in the absence of Tate tunings of gauge symmetries $\mathrm{SU}(n), n \geq 6$ and $\mathrm{SO}(n), n \geq 13$.
} 

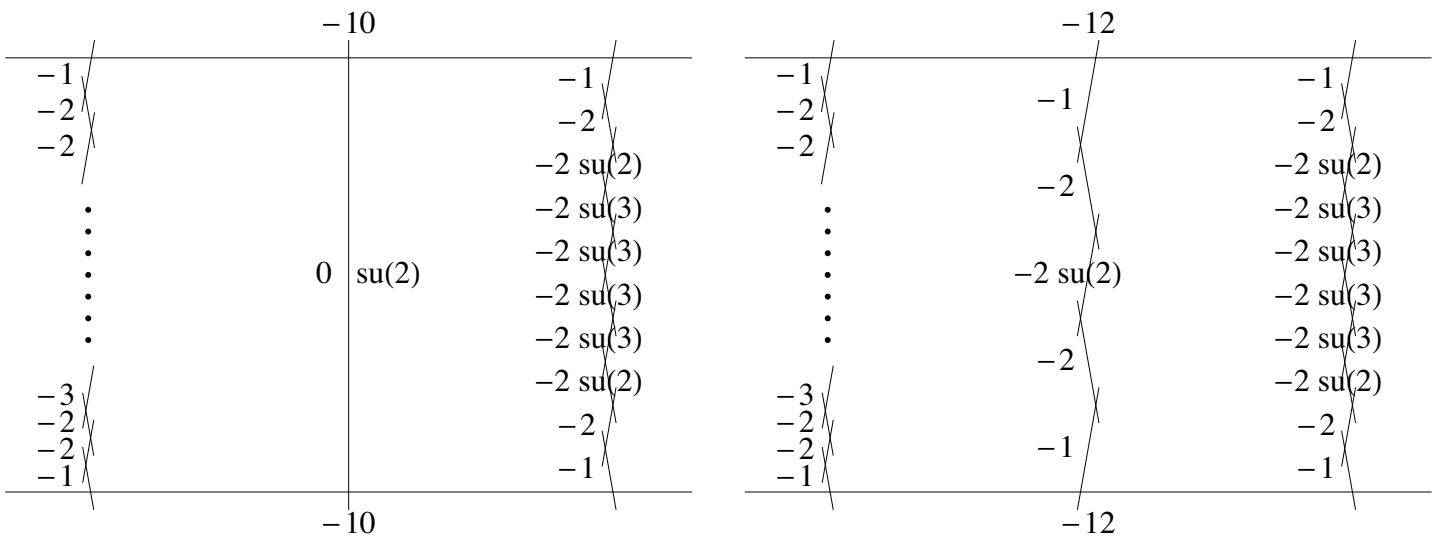

Figure 5. Base resolution that gives rise to a tuned flat elliptically fibered CY. Left: before resolution. Right: after resolving $(4,6)$ points in the base. The top curve is $D_{1}$ and the down curve is $D_{49}$. The curves in the left chain from top to down are $\left\{D_{2}, D_{2}, \ldots, D_{48}\right\}$, in the right chain from bottom to top are $\left\{D_{50}, D_{51}, \ldots, D_{59}\right\}$.

two lattice points $(0,0,0,1)$ and $(1,0,0,1)$ in $(4.13)$ contribute to the $x^{3}$ terms. The set of rays in the dual base $\tilde{B}$ is given by equation (4.3), and in this case

$$
\cup_{n \in\{1,2,3,4,6\}} V^{B}\left(a_{n}\right)=V^{B}\left(a_{4}\right) \cup V^{B}\left(a_{6}\right),
$$

which gives a 2D toric fan with the self-intersection numbers

$$
\tilde{B} \rightarrow[[-10 / /-12 / /-11 / /-12 / /-10,-1,-2,-2,-2,-2,-2,-2,-2,-2,-1]] .
$$

The generic elliptic fibration over $\tilde{B}$ has Hodge numbers $(143,23)$, so $\tilde{X}$ is a tuned model. As $\Delta$ is not a standard stacking $\mathbb{P}^{2,3,1}$ polytope, we do not have a Weierstrass model from a Tate form for this polytope. Nonetheless, the Weierstrass model can be obtained with the trick of "treating $\Delta$ as a $\mathrm{Bl}_{2} \mathrm{P}^{1,1,2}$-fibered polytope" as described in section 4.2 in [15]. This is a tuned Weierstrass model with tunings of gauge symmetries $\mathfrak{s u}(2) \oplus \mathfrak{s u}(3) \oplus \mathfrak{s u}(3) \oplus$ $\mathfrak{s u}(3) \oplus \mathfrak{s u}(3) \oplus \mathfrak{s u}(2)$ enhanced on $-2,-2,-2,-2,-2,-2\left(D_{52}^{(B)}-D_{57}^{(B)}\right)$, and also an $\mathfrak{s u}(2)$ gauge symmetry enhanced on a non-toric 0 -curve intersecting the two -10 -curves. The polytope $\Delta$ gives a non-flat elliptic fibration model; we can however find an equivalent flat elliptic fibration description with the same tuning of gauge symmetries over a resolved base [15]. In the resolved base, the original -10-curves are resolved to -12-curves through four successive blowups, and the non-toric 0 -curve is replaced by curves $-1,-2,-2,-2,-1$ where the two -1-curves intersect with the two -12-curves, respectively (see figure 5). The $\mathrm{SU}(2)$ gauge symmetry that was enhanced on the non-toric 0-curve is now enhanced on the middle -2 -curve in the blowup sequence; this is therefore a flat elliptic fibration. The Hodge number shifts of the flat fibration model calculated from the tunings match exactly with the polytope model: $(11,-16)+(1,-5)=(154,7)-(143,23)$.

\subsection{Other toric fibers (example: vertex stacking on fiber $F_{2}=\mathbb{P}^{1} \times \mathbb{P}^{1}$ )}

For the other examples we have considered so far we have restricted attention to fibrations with the fiber $F_{10}$ and the "standard stacking" form. The mirror symmetry structure also 
factorizes with other fiber types, though the physics of the corresponding F-theory models is more complicated and does not follow from standard Tate tuning structures. We give one example here of another toric fiber type, and leave further exploration of mirror symmetry with other fiber structures to further work.

As a simple example of a mirror pair of elliptically fibered CY3s associated with fibered polytopes with different fiber types, we start with an $F_{2}$-fibered polytope with base $B=\mathbb{P}^{2}$. We again use the "stacked" form where all the rays of the fan of $B$ are embedded within $\nabla$ in the form $\left(v_{i}^{B} ; v_{s}\right), v_{s} \in \nabla_{2}$. Let the stacking point be one of the $F_{2}$ vertices $v_{s}=(1,0)$. Therefore, the polytope $\nabla$ has vertices

$$
\left\{v_{i}\right\}=\{(-1,-1 ; 1,0),(1,0 ; 1,0),(0,1 ; 1,0),(0,0 ; 0,1),(0,0 ; 0,-1),(0,0 ;-1,0)\} .
$$

This corresponds to the polytope given by the data M:117 $8 \mathrm{~N}: 86 \mathrm{H}: 4,94$ [-180] in the KS database.

The mirror polytope also has a simple structure. The dual polytope $\Delta$ has vertices

$$
\begin{aligned}
\left\{w_{i}\right\}= & \{(-2,-2 ; 1,1),(4,-2 ; 1,1),(-2,4 ; 1,1),(-2,4 ; 1,-1),(-2,-2 ; 1,-1) \\
& (4,-2 ; 1,-1),(0,0 ;-1,1),(0,0 ;-1,-1)\}
\end{aligned}
$$

The mirror fiber $\tilde{F}=F_{15}$ has the vertices ${ }^{16}$

$$
\{(0,0,-1,-1),(0,0,1,-1),(0,0,1,1),(0,0,-1,1)\} \text {. }
$$

Over the points in the mirror fiber, we have points associated with the monomials in $\mathcal{O}\left(-2 K_{B}\right)$ over all the points $(1, y), y=-1,0,1$, and points associated with $\mathcal{O}\left(-K_{B}\right)$ over the points $(0, y), y=-1,0,1$, and only the points $(0,0 ;-1, y)$ over the remaining points in the fiber. From this we see that the dual base $\tilde{B}$ is given by the toric surface with the self-intersection sequence that can be read off from $\mathcal{O}\left(-2 K_{B}\right)$,

$$
\tilde{B} \rightarrow[[-1,-4,-1,-3,-1,-4,-1,-4,-1,-3,-1,-4,-1,-4,-1,-3,-1,-4]] .
$$

While the factorization of mirror symmetry is equally clear in this example to the others considered here, the F-theory interpretation is more subtle. A full analysis involves considerations using methods like those of $[21,25]$. We outline the analysis on the $\nabla$ side in this case and leave further work in this direction to the future. The polytope $\nabla$ has the single obvious fiber $F_{2}$, which does not provide a section, so this is a genus one fibration. We can analyze the gauge group and matter structure of the corresponding Jacobian fibration, which is relevant for F-theory [37]. The Weierstrass model of the Jacobian fibration has no nonabelian gauge symmetries. From the Hodge numbers we expect a nontrivial MordellWeil group of rank 2,

$$
G=\mathrm{U}(1) \times \mathrm{U}(1) .
$$

\footnotetext{
${ }^{16}$ Note that $\Delta$ has many distinct fibrations; the numbers of each of the 16 fiber types of $\Delta$ are $\{6,0,6,6,6,6,0,12,6,9,0,0,9,6,4,1\}$. We can immediately read off the mirror fibration however from the form of $\nabla$, and none of the other fibrations has a corresponding projection since the fiber of $\nabla$ is unique.
} 
Codimension two singularities in the Weierstrass model suggest $36+72+72$ matter fields charged in various ways under the $\mathrm{U}(1)$ factors in $G$, which is in accordance with the expected Hodge numbers $(4,94)=(2,272)+(2,2-(36+72+72))) \cdot{ }^{17}$

\subsection{Elliptic fibration with a non-fibered mirror}

As a final Calabi-Yau threefold example we consider a case where a CY threefold has an elliptic fibration associated with a $2 \mathrm{D}$ reflexive subpolytope, but the mirror has no fibration. In such cases, there cannot be a projection onto the fiber of $\Delta$, as discussed in section 2.2.

We consider the polytope from the KS database associated with the Calabi-Yau threefold with Hodge numbers $\left(h^{1,1}, h^{2,1}\right)=(149,1)$. There is a coordinate system in which this polytope $\nabla$ has vertices

$$
\left\{v_{i}\right\}=\{(0,0 ; 1,0),(-2,8 ;-3,-2),(-2,0 ;-3,-2),(6,0 ;-3,-2),(-2,-8,5,6)\} .
$$

In this coordinate system there is an $F_{10}$ reflexive 2 D fiber in the standard form $\left(0,0 ; v^{F}\right)$. The polytope does not satisfy the condition needed for the mirror to have a fibration, however, since the last vertex $v=\left(v^{(I)} ; v^{(I I)}\right), v^{(I I)}=(5,6)$ does not satisfy $v^{(I I)} \in \nabla_{2}$. Furthermore, it is straightforward to see that no linear transformation that preserves $\nabla_{2}$ can move all the vertices to satisfy this condition. In particular, the second and last vertices will always have $v^{(I I)}$ values that differ by a vector of the form

$$
v_{5}^{(I I)}-v_{2}^{(I I)}=(8,8)+16(x, y), x, y \in \mathbb{Z} .
$$

Thus, the mirror can never satisfy the fibration condition. This is not surprising since the mirror has Hodge numbers $(1,149)$ and cannot have a genus one or elliptic fibration since the Shioda-Tate-Wazir would give $h^{1,1}(\tilde{X}) \geq h^{1,1}(\tilde{B})+1 \geq 2$. One can also check explicitly that the lattice points in the mirror polytope $\Delta$ do not contain a linearly embedded $\mathbb{P}^{2,3,1}$ fiber.

\subsection{Elliptic Calabi-Yau fourfolds (example: generic elliptic fibration over $\mathbb{P}^{3}$ )}

The factorization structure that we have described for elliptic toric hypersurface CalabiYau threefolds can occur in much the same fashion for higher-dimensional Calabi-Yau manifolds realized as toric hypersurfaces. We leave a more detailed investigation of higherdimensional Calabi-Yau mirror symmetry for future work, but note here simply that the simplest cases of generic elliptic fibrations over toric bases have a natural generalization to arbitrary higher dimension. We present here only a single simple case, the Calabi-Yau fourfold given by the generic elliptic fibration over the base $\mathbb{P}^{3}$. The polytope $\nabla$ in this case is the simple generalization of the polytope with vertices given by eq. (3.2), and has vertices

$$
\begin{aligned}
\left\{v_{i}\right\}= & \{(0,0,0 ; 1,0),(0,0,0 ; 0,1),(1,0,0 ;-3,-2),(0,1,0 ;-3,-2), \\
& (0,0,1 ;-3,-2),(-1,-1,-1 ;-3,-2)\} .
\end{aligned}
$$

\footnotetext{
${ }^{17}$ Note that from [21], the F-theory models associated with the Jacobian fibrations of $F_{2}$ fibered polytopes should generically have toric Mordell-Weil group $\mathrm{U}(1) \times \mathbb{Z}_{2}$. The stacked form we have here over a vertex of $F_{2}$ gives a degeneration that may enhance the $\mathbb{Z}_{2}$ to a $U(1)$ factor. (Thanks to Paul Oehlmann for explaining this to us.)
} 
The Hodge numbers of the corresponding Calabi-Yau fourfold are $h^{1,1}(X)=2, h^{3,1}(X)=$ 3878. The dual polytope $\Delta$ is again $\mathbb{P}^{2,3,1}$ fibered, with a base $\tilde{B}$ given by the set of toric rays within the polytope having vertices

$$
\left\{v_{i}^{(\tilde{B})}\right\}=\{(-6,-6,-6),(18,-6,-6),(-6,18,-6),(-6,-6,18)\} .
$$

There are a tremendous number of ways of triangulating this $5 \mathrm{D}$ polytope to give a complete toric fan. Independent of the triangulation, however, the generic elliptic fibration over the toric base $\tilde{B}$ will have a non-Higgsable gauge group

$$
G=E_{8}^{34} \times F_{4}^{96} \times G_{2}^{256} \times \mathrm{SU}(2)^{384} .
$$

This can be seen from the numbers of primitive rays in $\tilde{B}$ with specific minimum values of the inner products with the vertices in (4.23). For each triangulation, the mirror CalabiYau will have Hodge numbers $h^{1,1}(\tilde{X})=3878, h^{3,1}(\tilde{X})=2$. An interesting feature of the mirror Calabi-Yau fourfolds associated with the polytope $\Delta$ is that it is one of the "attractive" endpoints reached by blowing up $\mathbb{P}^{3}$ as far as possible consistent with a base that supports an elliptic fibration [38]. This may be associated with the large number of triangulations that are possible in this case. The other attractive endpoints also are factorizable mirrors over simple bases that are $3 \mathrm{D}$ toric varieties with few rays. We leave further exploration of the many interesting questions associated with mirror symmetry and Calabi-Yau fourfolds to further work.

\section{Conclusions and further questions}

\subsection{Summary of results}

Building on recent work [16] in which we showed that most toric hypersurface Calabi-Yau threefolds have a manifest genus one or elliptic fibration, we have found that many of these elliptic fibrations exhibit a mirror symmetry that factorizes, in the sense that the fiber $F$ of $X$ is a mirror Calabi-Yau 1-fold to the fiber $\tilde{F}$ of $\tilde{X}$. This connects with a number of directions of earlier research related to aspects of such mirror fibers [17-21, 39, 40]. We have furthermore found that the structure of the mirror base $\tilde{B}$ is determined in a clear and well-defined way from the base $B$ and fibration structure of $X$. In many cases of interest the mirror base takes a simple form in terms of the toric geometry of a line bundle over $B$. In particular, for generic CY elliptic fibrations over toric base surfaces, the mirror base $\tilde{B}$ has a toric fan that is built from the primitive rays in the set of sections of the line bundle $\mathcal{O}\left(-6 K_{B}\right)$. For tuned Tate models over a toric base surface, there is a slightly more complicated expression for the rays in $\tilde{B}$, given by (4.1) when $\Delta$ is also a standard stacking polytope, or (4.3) when $\Delta$ fails to maintain the standard stacking structure due to excessive removal of points associated with monomials in $\mathcal{O}\left(-6 K_{B}\right)$. As shown in [15], almost all the Hodge number pairs in the KS database with $h^{1,1} \geq 240$ or $h^{2,1} \geq 240$ are realized by generic or tuned elliptic fibrations with the standard stacking structure.

We have explored some simple examples of this factorized mirror symmetry, particularly for some cases of Calabi-Yau threefolds with large Hodge numbers, and generic and tuned elliptic fibrations over simple bases such as $\mathbb{P}^{2}$ and the Hirzebruch surfaces $\mathbb{F}_{n}$. 
With growing evidence that most known Calabi-Yau threefolds admit a genus one or elliptic fibration, the results we have found here suggest that there may be a very general way of understanding mirror symmetry in terms of fibration by smaller-dimensional CalabiYau fibers. Further work is clearly needed to explore the details of the mirror dictionary for different bases and fibers, in higher dimensions, and the extent to which the factorization structure identified here can be extended beyond the toric hypersurface framework.

\subsection{Further questions and directions}

We list here some specific open questions that may be of interest for further research.

- We have given a variety of examples here where mirror symmetry between a pair of elliptic Calabi-Yau threefolds factorizes between the base and the fiber of the fibrations. From these examples it is clear that there are regular local structures that could be used to begin to form a dictionary relating structure on the mirror base $\tilde{B}$ to structure of the base $B$ and fibration structure of the original elliptic Calabi-Yau $X$. For example, a curve of self-intersection +1 , such as was encountered in the example in section 3.2, naturally corresponds to a sequence of toric curves of self-intersections $/ /-12 / /-12 / /$ in the mirror base $\tilde{B}$. Similarly, in table 2 , we see a pattern where blowing up a non-toric vs. toric point in the base $B$ corresponds to blowing up a toric vs. non-toric point in the mirror base $\tilde{B}$. It would be interesting to try to systematically develop this kind of structure, ideally including the additional reductions on the base $\tilde{B}$ that are imposed by different Tate tunings on the generic elliptic fibration over a toric $B$, which can be understood through additional constraints arising from the associated "tops".

- We know that at large Hodge numbers many of the Calabi-Yau threefolds in the Kreuzer-Skarke database are generic or tuned elliptic fibrations over toric bases that can be constructed from polytopes with a fiber $F_{10}$, and these exhibit the simplest forms of mirror factorization studied in section 3, section 4 . It would be interesting to study further what fraction of the KS database exhibit mirror factorization and what other types of structures arise frequently or in isolated cases at smaller Hodge numbers.

- We have focused here primarily on the simplest cases where the toric $2 \mathrm{D}$ fiber is the self-dual fiber $F_{10}$, corresponding to generic elliptic fibrations. It would be interesting to study in more detail the structure of the other $2 \mathrm{D}$ toric fibers. In particular, it was found in [21] that the dual fibers $F_{i}, \tilde{F}_{i}$ exhibit some interesting structure, including identical numbers of sections associated with toric Mordell-Weil rank, and a matching between Mordell-Weil torsion on one side and discrete symmetries associated with the Tate-Shafarevich/Weil-Chatalet group on the dual side. It would be interesting to understand better how these features of the fibers can be used in understanding mirror symmetry of the full Calabi-Yau threefolds $X, \tilde{X}$ with the different dual fiber types.

- One natural way of trying to extend the analysis here is to look at complete intersection Calabi-Yau varieties. A large class of complete intersection fibers were analyzed 
in [39], and the properties of mirror fibers in these more general cases were noted in this paper and studied more thoroughly in [40]. It would be interesting to investigate these structures further in the context of full elliptic Calabi-Yau threefolds (and fourfolds).

- One of the most powerful approaches to mirror symmetry that has been used in earlier work is the Strominger-Yau-Zaslow (SYZ) picture [41], in which mirror symmetry is realized by T-duality on a 3-torus fiber over a real threefold base. While this picture has led to some powerful insights into mirror symmetry, it is incompatible with the algebro-geometric structure of Calabi-Yau manifolds, and the 3-torus fibration of a general Calabi-Yau is extremely singular. The factorization structure identified here seems to match more naturally with ideas from algebraic geometry and involve more controlled singularity structures. It would be interesting to understand whether there is a way of relating the SYZ picture to the factorization structure found here.

- As described in a simple example in section 4.6, the factorization structure explored here should be equally valid for higher-dimensional Calabi-Yau varieties, and particularly for Calabi-Yau fourfolds. It would be interesting to explore further the structures that arise for mirror symmetry of elliptic Calabi-Yau fourfolds.

- The mirror symmetry identified here between elliptic fibrations suggests that there may be an interesting corresponding duality in F-theory. This would be interesting to explore further.

- It would be interesting to connect this factorization structure of mirror symmetry to other aspects of mirror symmetry research and Calabi-Yau geometry. For example, it would be interesting to understand how the form of Calabi-Yau periods and the structure of the moduli space, or recent progress on the all genus amplitudes of topological string theory on elliptic Calabi-Yau threefolds [42] fits into this factorized picture.

- There are several contexts in which this structure of mirror symmetry may have an interesting physical interpretation or consequences for physics. Beyond the possibility mentioned above of a new duality in F-theory, for more conventional type II mirror symmetry the existence of an elliptic fibration on both sides of the duality suggests additional structure that may lead to physical insights. We have not explored this direction significantly in this paper but it should be a very interesting direction for further study.

\section{Acknowledgments}

Particular thanks to Yinan Wang for insight and discussions related to this work; as mentioned in section 3 , the simplest class of examples of the mirror symmetry structure explored here were identified some years earlier by Wang in the context of a separate project with Andreas Braun [32]. We would also like to thank Lara Anderson, Andreas Braun, Antonella Grassi, James Gray, Sheldon Katz, Albrecht Klemm, Dave Morrison, Paul Oehlmann, and 
Andrew Turner for helpful discussions. This material is based upon work supported by the U.S. Department of Energy, Office of Science, Office of High Energy Physics under grant Contract Number DE-SC00012567.

\section{A The 16 reflexive $2 \mathrm{D}$ fiber polytopes $\nabla_{2}$}

We list here the 16 reflexive $2 \mathrm{D}$ polytopes $\nabla_{2}$. The mirror of fiber $F=F_{i}$ is the fiber $\tilde{F}=F_{17-i}$ for $i<7, i>10$; the fibers $F_{i}$ for $i=7,8,9,10$ are self-mirror up to linear transformations. For each lattice point $v^{F}$, we include the maximum value of $1+v^{F}$. $m^{(I I)}, m^{(I I)} \in \Delta_{2}$.
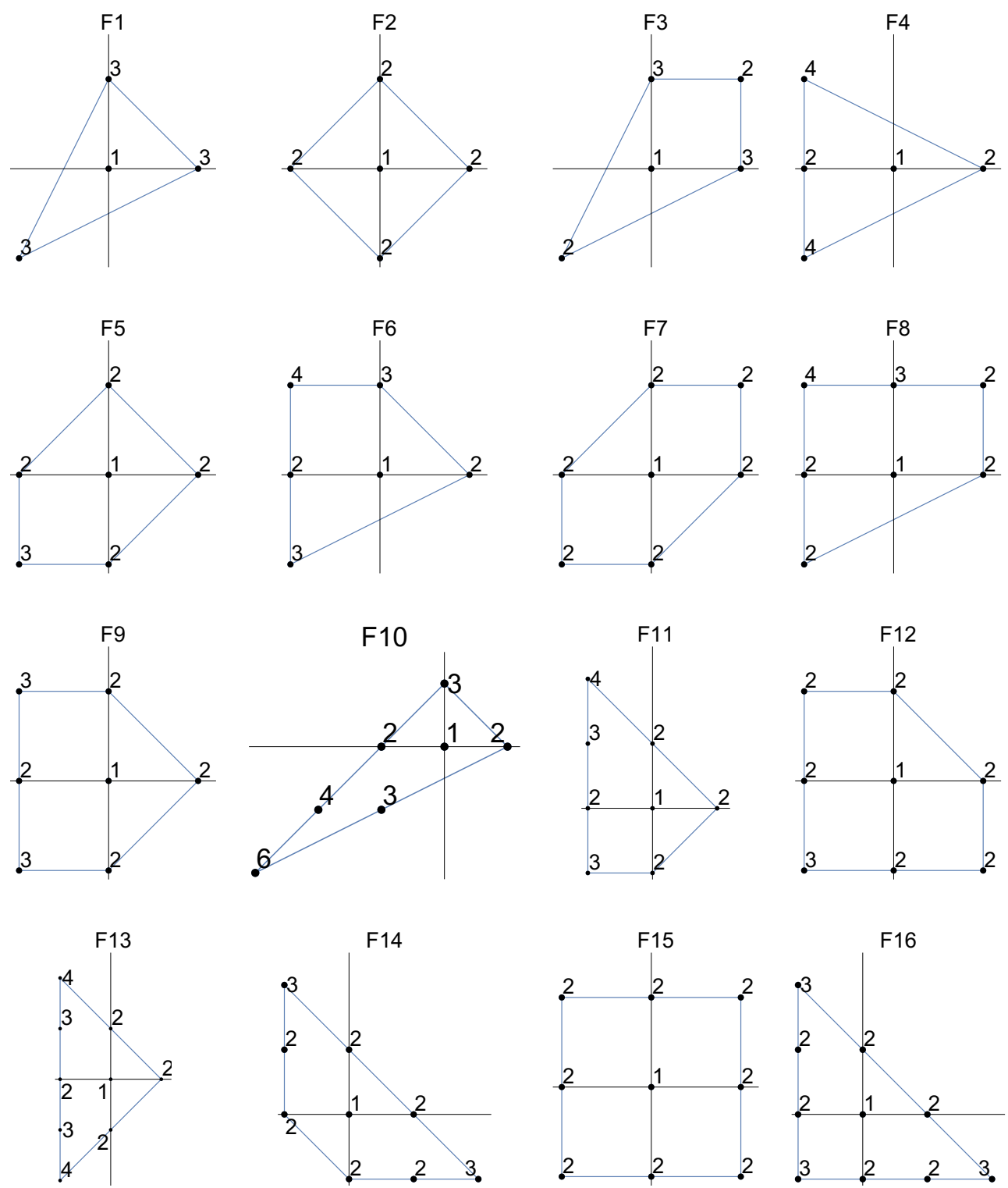


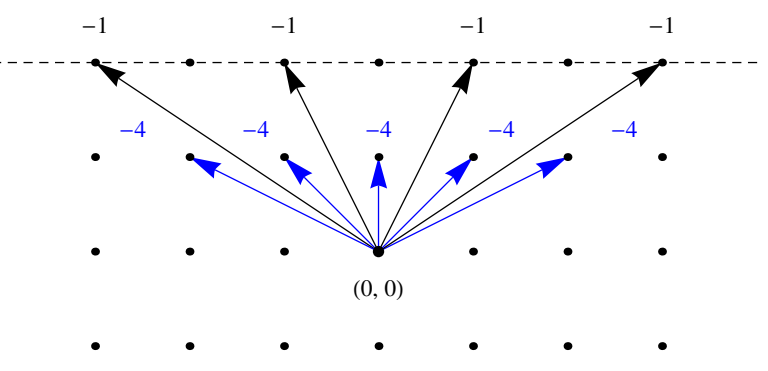

Figure 6. Primitive rays associated with a face of the toric base polytope at distance 2 from the origin give a sequence of toric curves of self-intersection $-1,-4,-1,-4, \ldots$.

\section{B Faces of the base polytope and chains of non-Higgsable clusters}

Certain chains of self-intersections of curves in the base, associated with characteristic combinations of non-Higgsable clusters, have been observed both in $6 \mathrm{D}$ supergravity theories [31, 33] and 6D superconformal field theories constructed from F-theory [34, 43, 44]. In the context of the toric bases we consider here, these can be seen as arising simply from the sequences of primitive rays in a toric base associated with a face of the bounding polytope at different distances from the origin. We encounter the $E_{8}$ sequence connecting -12 curves in the example in section 3.2 , and the simple $\mathrm{SO}(8)$ sequence connecting -4 curves in the example in section 4.4. We briefly discuss here all the constructions of this type to illustrate how they arise in a unifying context in this framework.

The simplest case is that of the self-intersection sequence $[[-1,-4,-1,-4,-1, \ldots]]$. This can be seen as arising from the set of primitive rays associated with a face of the toric base polytope at distance 2 from the origin (figure 6 ). The primitive rays in this case are of the forms $(n, 1) \forall n$ and $(2 k+1,2) \forall k$. For example, starting from $(0,1)$, the sequence of rays in a toric diagram associated with a polytope having a face along the line $(x, 2)$ is

$$
(0,1),(1,2),(1,1),(3,2),(2,1), \ldots
$$

Since from toric geometry we know that a toric curve $v_{i}$ has self-intersection $-n$ when $n v_{i}=v_{i-1}+v_{i+1}$, we can read off the self-intersection sequence $[[-4,-1,-4,-1,-4, \ldots]]$ from the ray sequence (B.1). This corresponds to a sequence of non-Higgsable gauge groups $\mathrm{SO}(8), \cdot, \mathrm{SO}(8), \cdot, \mathrm{SO}(8) \ldots$ in the F-theory picture.

Performing a similar analysis at other distances we see that the following sequences arise:

$$
\begin{aligned}
d=2 \rightarrow & {[[-4,-1,-4, \ldots]](\mathrm{SO}(8)) } \\
d=3 \rightarrow & {[[-6,-1,-3,-1,-6, \ldots]]\left(E_{6} \times \mathrm{SU}(3)\right) } \\
d=4 \rightarrow & {[[-8,-1,-2,-3,-2,-1,-8, \ldots]]\left(E_{7} \times(\mathrm{SU}(2) \times \mathrm{SO}(7) \times \mathrm{SU}(2))\right) } \\
d=6 \rightarrow & {[[-12,-1,-2,-2,-3,-1,-5,-1,-3,-2,-2,-1,-12]] } \\
& \left(E_{8} \times F_{4} \times\left(G_{2} \times \mathrm{SU}(2)\right)^{2}\right)
\end{aligned}
$$

where in each case the sequence repeats and we have indicated the non-Higgsable gauge group for a single cycle of the sequence. These are precisely the maximal connected sequences identified in [33] and associated with e.g. " $E_{8}$ matter" in [34]. 
Open Access. This article is distributed under the terms of the Creative Commons Attribution License (CC-BY 4.0), which permits any use, distribution and reproduction in any medium, provided the original author(s) and source are credited.

\section{References}

[1] P. Candelas, G.T. Horowitz, A. Strominger and E. Witten, Vacuum Configurations for Superstrings, Nucl. Phys. B 258 (1985) 46 [InSPIRE].

[2] K. Hori et al. eds., Clay Mathematics Monographs. Vol. 1: Mirror Symmetry, AMS Press, Providence U.S.A. (2003).

[3] P. Candelas, M. Lynker and R. Schimmrigk, Calabi-Yau Manifolds in Weighted P(4), Nucl. Phys. B 341 (1990) 383 [inSPIRE].

[4] V.V. Batyrev, Dual polyhedra and mirror symmetry for Calabi-Yau hypersurfaces in toric varieties, J. Alg. Geom. 3 (1994) 493 [alg-geom/9310003] [INSPIRE].

[5] M. Kreuzer and H. Skarke, Complete classification of reflexive polyhedra in four-dimensions, Adv. Theor. Math. Phys. 4 (2002) 1209 [hep-th/0002240] [INSPIRE].

[6] M. Kreuzer and H. Skarke, http://hep.itp.tuwien.ac.at/ kreuzer/CY.html.

[7] W. Taylor, On the Hodge structure of elliptically fibered Calabi-Yau threefolds, JHEP 08 (2012) 032 [arXiv: 1205.0952] [INSPIRE].

[8] P. Candelas, A. Constantin and H. Skarke, An Abundance of K3 Fibrations from Polyhedra with Interchangeable Parts, Commun. Math. Phys. 324 (2013) 937 [arXiv:1207.4792] [INSPIRE].

[9] J. Gray, A.S. Haupt and A. Lukas, Topological Invariants and Fibration Structure of Complete Intersection Calabi-Yau Four-Folds, JHEP 09 (2014) 093 [arXiv:1405.2073] [INSPIRE].

[10] S.B. Johnson and W. Taylor, Calabi-Yau threefolds with large $h^{2,1}$, JHEP 10 (2014) 23 [arXiv:1406.0514] [INSPIRE].

[11] L.B. Anderson, X. Gao, J. Gray and S.-J. Lee, Tools for CICYs in F-theory, JHEP 11 (2016) 004 [arXiv: 1608. 07554] [INSPIRE].

[12] L.B. Anderson, X. Gao, J. Gray and S.-J. Lee, Multiple Fibrations in Calabi-Yau Geometry and String Dualities, JHEP 10 (2016) 105 [arXiv: 1608.07555] [INSPIRE].

[13] L.B. Anderson, X. Gao, J. Gray and S.-J. Lee, Fibrations in CICY Threefolds, JHEP 10 (2017) 077 [arXiv : 1708.07907] [inSPIRE].

[14] L.B. Anderson, J. Gray and B. Hammack, Fibrations in Non-simply Connected Calabi-Yau Quotients, JHEP 08 (2018) 128 [arXiv: 1805.05497] [INSPIRE].

[15] Y.-C. Huang and W. Taylor, Comparing elliptic and toric hypersurface Calabi-Yau threefolds at large Hodge numbers, JHEP 02 (2019) 087 [arXiv: 1805.05907] [INSPIRE].

[16] Y.-C. Huang and W. Taylor, On the prevalence of elliptic and genus one fibrations among toric hypersurface Calabi-Yau threefolds, JHEP 03 (2019) 014 [arXiv:1809.05160] [INSPIRE].

[17] A.C. Avram, M. Kreuzer, M. Mandelberg and H. Skarke, Searching for K3 fibrations, Nucl. Phys. B 494 (1997) 567 [hep-th/9610154] [INSPIRE]. 
[18] P. Berglund and P. Mayr, Heterotic string/F theory duality from mirror symmetry, Adv. Theor. Math. Phys. 2 (1999) 1307 [hep-th/9811217] [INSPIRE].

[19] A. Grassi and V. Perduca, Weierstrass models of elliptic toric K3 hypersurfaces and symplectic cuts, Adv. Theor. Math. Phys. 17 (2013) 741 [arXiv:1201.0930] [inSPIRE].

[20] M. Cvetič, A. Grassi and M. Poretschkin, Discrete Symmetries in Heterotic/F-theory Duality and Mirror Symmetry, JHEP 06 (2017) 156 [arXiv:1607.03176] [INSPIRE].

[21] D. Klevers, D.K. Mayorga Pena, P.-K. Oehlmann, H. Piragua and J. Reuter, F-Theory on all Toric Hypersurface Fibrations and its Higgs Branches, JHEP 01 (2015) 142 [arXiv: 1408.4808] [INSPIRE].

[22] V. Batyrev, Variations of the mixed Hodge structure of affine hypersurfaces in algebraic tor, Duke Math. J. 69 (1993) 349.

[23] M. Kreuzer and H. Skarke, Calabi-Yau four folds and toric fibrations, J. Geom. Phys. 26 (1998) 272 [hep-th/9701175] [INSPIRE].

[24] F. Rohsiepe, Fibration structures in toric Calabi-Yau fourfolds, hep-th/0502138 [INSPIRE].

[25] V. Bouchard and H. Skarke, Affine Kac-Moody algebras, CHL strings and the classification of tops, Adv. Theor. Math. Phys. 7 (2003) 205 [hep-th/0303218] [InSPIRE].

[26] V. Braun, Toric Elliptic Fibrations and F-theory Compactifications, JHEP 01 (2013) 016 [arXiv:1110.4883] [INSPIRE].

[27] V. Braun, T.W. Grimm and J. Keitel, Geometric Engineering in Toric F-theory and GUTs with U(1) Gauge Factors, JHEP 12 (2013) 069 [arXiv:1306.0577] [INSPIRE].

[28] H. Skarke, String dualities and toric geometry: An Introduction, Chaos Solitons Fractals 10 (1999) 543 [hep-th/9806059] [INSPIRE].

[29] A.P. Braun and M. Del Zotto, Mirror Symmetry for $G_{2}$-Manifolds: Twisted Connected Sums and Dual Tops, JHEP 05 (2017) 080 [arXiv:1701.05202] [INSPIRE].

[30] P. Candelas and A. Font, Duality between the webs of heterotic and type-II vacua, Nucl. Phys. B 511 (1998) 295 [hep-th/9603170] [INSPIRE].

[31] D.R. Morrison and W. Taylor, Toric bases for 6D F-theory models, Fortsch. Phys. 60 (2012) 1187 [arXiv: 1204.0283] [INSPIRE].

[32] A. Braun, W. Taylor and Y. Wang, unpublished notes (2016).

[33] D.R. Morrison and W. Taylor, Classifying bases for $6 D$ F-theory models, Central Eur. J. Phys. 10 (2012) 1072 [arXiv:1201.1943] [INSPIRE].

[34] M. Del Zotto, J.J. Heckman, A. Tomasiello and C. Vafa, 6d Conformal Matter, JHEP 02 (2015) 054 [arXiv: 1407.6359] [INSPIRE].

[35] D.R. Morrison and C. Vafa, Compactifications of F-theory on Calabi-Yau threefolds. 1, Nucl. Phys. B 473 (1996) 74 [hep-th/9602114] [INSPIRE].

[36] D.R. Morrison and C. Vafa, Compactifications of F-theory on Calabi-Yau threefolds. 2, Nucl. Phys. B 476 (1996) 437 [hep-th/9603161] [INSPIRE].

[37] D.R. Morrison and W. Taylor, Sections, multisections and U(1) fields in F-theory, arXiv: 1404.1527 [INSPIRE].

[38] W. Taylor and Y.-N. Wang, Scanning the skeleton of the 4D F-theory landscape, JHEP 01 (2018) 111 [arXiv:1710.11235] [INSPIRE]. 
[39] V. Braun, T.W. Grimm and J. Keitel, Complete Intersection Fibers in F-theory, JHEP 03 (2015) 125 [arXiv:1411.2615] [INSPIRE].

[40] P.-K. Oehlmann, J. Reuter and T. Schimannek, Mordell-Weil Torsion in the Mirror of Multi-Sections, JHEP 12 (2016) 031 [arXiv:1604.00011] [INSPIRE].

[41] A. Strominger, S.-T. Yau and E. Zaslow, Mirror symmetry is T duality, Nucl. Phys. B 479 (1996) 243 [hep-th/9606040] [INSPIRE].

[42] M.-x. Huang, S. Katz and A. Klemm, Topological String on elliptic CY 3-folds and the ring of Jacobi forms, JHEP 10 (2015) 125 [arXiv:1501.04891] [INSPIRE].

[43] J.J. Heckman, D.R. Morrison and C. Vafa, On the Classification of 6D SCFTs and Generalized ADE Orbifolds, JHEP 05 (2014) 028 [Erratum ibid. 1506 (2015) 017] [arXiv: 1312.5746] [INSPIRE].

[44] N. Mekareeya, K. Ohmori, H. Shimizu and A. Tomasiello, Small instanton transitions for M5 fractions, JHEP 10 (2017) 055 [arXiv:1707.05785] [INSPIRE]. 